Silvio Dutz*, Andreas Weidner, Moritz von der Lühe, Christine Gräfe, Philip Biehl, Johanna Demut, Paul Warncke, Sandra Jungmann, Dagmar Fischer, Felix H. Schacher and Joachim H. Clement

\title{
6 Hybrid nanomaterials of biomolecule corona coated magnetic nanoparticles and their interaction with biological systems
}

\begin{abstract}
Magnetic nanoparticles (MNPs) are interesting for various applications in medicine. If administered to a biological system like the human body, a so-called biomolecule corona is formed on the surface of the particles, which highly determines the biological fate of the particles. To elucidate whether a preconditioning of the MNPs by incubation with biomolecules influences biocompatibility and bioavailability, the formation of such a corona was investigated in more detail. For this, the influence of particle characteristics, e.g., surface charge, as well as various incubation parameters on the resulting corona was investigated. It was found that the biomolecule corona is
\end{abstract}

\begin{abstract}
*Corresponding author: Silvio Dutz, Institute of Biomedical Engineering and Informatics (BMTI), Technische Universität Ilmenau, Ilmenau, Germany; and Department of Nano Biophotonics, Leibniz Institute of Photonic Technology (IPHT), Jena, Germany, e-mail: silvio.dutz@tu-ilmenau.de, https:// orcid.org/0000-0002-7258-0943
\end{abstract}

Andreas Weidner, Institute of Biomedical Engineering and Informatics (BMTI), Technische Universität Ilmenau, Ilmenau, Germany, e-mail: Andreas.Weidner@tu-ilmenau.de

Moritz von der Lühe, Philip Biehl and Felix H. Schacher, Institute of Organic Chemistry and Macromolecular Chemistry (IOMC), Friedrich-Schiller-University Jena, Jena, Germany; and Jena Center for Soft Matter (JCSM), Friedrich-Schiller-University Jena, Jena, Germany, e-mail: moritz.von-derluehe@uni-jena.de (M. von der Lühe), philip.biehl@uni-jena.de (P. Biehl), felix.schacher@uni-jena.de (F.H. Schacher)

Christine Gräfe and Johanna Demut, Klinik für Innere Medizin II, Abteilung Hämatologie und Internistische Onkologie, Universitätsklinikum Jena, Jena, Germany, e-mail: christine.graefe@med.uni-jena.de (C. Gräfe), Johanna.Demut@med.uni-jena.de (J. Demut) Paul Warncke and Sandra Jungmann, Institute of Pharmacy, Pharmaceutical Technology und Biopharmacy, Friedrich-Schiller-University Jena, Jena, Germany, e-mail: paul.warncke@uni-jena.de (P. Warncke), sandra.jungmann@uni-jena.de (S. Jungmann)

Dagmar Fischer, Jena Center for Soft Matter (JCSM), Friedrich-Schiller-University Jena, Jena, Germany; and Institute of Pharmacy, Pharmaceutical Technology und Biopharmacy, Friedrich-Schiller-University Jena, Jena, Germany, e-mail: dagmar.fischer@uni-jena.de

Joachim H. Clement, Jena Center for Soft Matter (JCSM), Friedrich-Schiller-University Jena, Jena, Germany; and Klinik für Innere Medizin II, Abteilung Hämatologie und Internistische Onkologie, Universitätsklinikum Jena, Jena, Germany, e-mail: Joachim.Clement@med.uni-jena.de. https:// orcid.org/0000-0002-6601-2456

D Open Access. (๑) 2020 Silvio Dutz et al., published by De Gruyter. (c) BY-NC-ND This work is licensed under the Creative Commons Attribution-NonCommercial-NoDerivatives 4.0 International License.

This article has previously been published in the journal Physical Sciences Reviews. Please cite as: S. Dutz, A. Weidner, M. von der Lühe, C. Gräfe, P. Biehl, J. Demut, P. Warncke, S. Jungmann, D. Fischer, F. H. Schacher and J. H. Clement "Hybrid nanomaterials of biomolecule corona coated magnetic nanoparticles and their interaction with biological systems" Physical Sciences Reviews [Online] 2020, 5. DOI: 10.1515/psr-2019-0110 | https://doi.org/10.1515/9783110569636-006 
formed immediately after bringing together the particles with the biomolecule source. By variation of the biomolecule content of the incubation medium, the size of the corona can be modulated. Regarding the interaction of the nanoparticles with cells, it was shown that the presence of a biomolecule corona reduces the interaction and that a more pronounced biomolecule corona leads to a reduced uptake of the magnetic nanohybrids into the cells. Cell viability tests confirmed biocompatibility of the biomolecule-coated particles. A more pronounced corona promotes a higher cell viability. By using a shell-less hen's egg model, no or reduced adverse effects of all biomolecule-coated MNP for this in vivo test were found. Resulting from these investigations, we were able to demonstrate that our newly developed nanohybrids significantly reduce in vivo toxicity compared to uncoated MNPs.

Keywords: biomolecule corona; hybrid materials; magnetic nanoparticles; protein corona.

\subsection{Introduction}

Magnetic nanoparticles (MNPs) and their biocompatible suspensions (ferrofluids) are very promising materials for biomedical applications [1-4]. Due to their high surfaceto-volume ratio and small size, MNPs exhibit properties differing from that of the bulk material, like superparamagnetism or stability against sedimentation. They possess magnetic properties that enable magnetic manipulation or heating by external magnetic fields, so that they cannot only be used in therapy, but also in diagnostics and as tracers. A wide range of applications like hyperthermia [5-7], drug targeting [2, 8, 9] or as contrast and tracer agent for medical imaging [10] are under development.

Therefore, a lot of hybrid MNP systems with specific cores and coatings have been developed and tested for different applications. Due to their high biocompatibility, especially iron oxide MNP is of great interest as a core material [11-13]. The so-called magnetic multicore nanoparticles (MCNPs) show superferrimagnetism, which means ferrimagnetic behavior in presence of an external magnetic field and superparamagnetic behavior in its absence. Such particles consist of clusters made of small single domain primary cores. Due to statistical orientation of easy axis of the primary cores within the clusters, the resulting magnetization without an external field is relatively low in comparison to single core particles in the size of the clusters. This means, these large particles show a very weak remnant magnetization like superparamagnetic particles und thus only a very low tendency to form agglomerates. This makes such particles relatively stable against sedimentation which is a good basis for medical applications. On the other hand, when exposed to an external magnetic field, these particles behave like bigger ferrimagnetic particles with a pronounced coercivity due to exchange interactions between primary particles. 
The MCNPs are very promising for medical application [14], especially for magnetic manipulation due to their large particle volume and for hyperthermia because of their high heating performance [15-17]. Superparamagnetic iron oxide nanoparticles (SPIONs) are of particular interest for application as tracer and contrast agents for magnetic particle imaging (MPI) and magnetic resonance imaging (MRI), respectively $[2,13,18]$. Typically, MNP of all different core types are covered by diverse coatings like inorganic shells (e.g., silica) or organic ones (e.g., dextrans or polymers) to prevent agglomeration, resulting in sedimentation stable fluids of the MNPs for medical application. Additionally, the coating serves as an anchor layer for functionalization with a pharmaceutical agent or dyes.

One of the main obstacles for the medical use of these promising materials is their behavior when applied to biological liquids and biological systems, like the human body. For decades chemists, biologists, and engineers have developed methods to stabilize the MNP in aqueous suspensions and test biocompatibility in vitro and in vivo. After application of MNP into a biological system, it was found that the MNPs are immediately covered by biomolecules available within the body. A so-called "biomolecule corona" [19-21] is formed that changes their behavior drastically and gives them a new biological identity [22]. This additional biomolecule coating, consisting mainly of a protein corona and lipids, directly influences the properties of the used MNP. The biomolecule corona alters surface chemistry and thus their stability against agglomeration and sedimentation [23-25], as well as their interaction with biological systems in vitro and in vivo $[19,26,27]$. To enable a safe and reliable administration of MNP to biological systems for medical reasons, the knowledge of the influencing factors on structure and amount of an evolving biomolecule corona and the possibilities to control and to include the corona formation as a positive event is of major importance.

A deeper understanding of the complex interactions of MNP with biomolecules and biological systems is still in progress [19, 28-31] and first promising results could be made based on the concept of a "hard" and "soft" corona [32]. While the "hard" corona describes the fact that several biomolecules are strongly adsorbed to the surface of the MNP and only slow changes of the composition can be observed, the "soft" corona changes dynamically within time and depending on the environment [30, 33, 34]. Due to its temporal stability, the "hard" corona can be investigated with established measurements regarding its size, shape, hydrodynamic size, surface charge, as well as methods to determine their composition and integrity [32, 35-38]. Due to their magnetic properties, the MNP enable the use of dynamic magnetic measurements, what gives additional information on particle-matrix interactions and can help to understand the dynamic process of corona formation and protein cross-linking in more detail [39-41].

In the recent research on corona formation, it was found that the corona formation and the composition and amount of the final "hard" corona is depending on key parameters like particle size, surface charge, temperature, or incubation time [20, 4248], what gives the possibility to shape, control and modify the corona and thus the 
particle-matrix interactions to avoid harmful effects like unwanted agglomeration or sedimentation and supporting beneficial effects like a longer blood half-life and lower toxicity [49-54].

That is why the main objectives of our work on this topic have been to develop and to show ways for reproducible preparation of sedimentation stable and biocompatible hybrid core-shell MNP (magnetic nanohybrid) suspensions for medical application.

Main approach to achieve these objectives was the pre-incubation of these MNP with different coatings in a biological fluid, serving as a source for the formation of the biomolecule corona under controlled conditions, to investigate the evolving biomolecule corona and to study the interactions of the magnetic nanohybrids with biological systems in vitro and in vivo. For this, we investigated and optimized MNP systems with different cores (MCNP, SPION) and different coatings featuring different surface charges and charge patterns (positive, negative, neutral, zwitterionic). We tested the influence of these parameters together with the parameters for the preincubation procedure on the resulting structure and amount of biomolecules bound to the surface of the MNP. Additionally, we investigated the interactions of these magnetic nanohybrids with biological systems like cells in established toxicity assays, as well as in quasi in vivo assays using the shell-less hen's egg test on the chick area vasculosa (HET-CAV). Furthermore, we developed strategies to sterilize and store the magnetic nanohybrids for a longer term, which is crucial towards their application in medicine. In this paper, we present a review of our main findings on these questions, obtained from our work in the Priority Program SPP1681 of the German Research Foundation Deutsche Forschungsgemeinschaft over the past six years [50, 55-62].

\subsection{Methods}

\subsubsection{Preparation of nanohybrids}

The magnetic nanohybrids used in this study mainly consist of a magnetic core of magnetic iron oxide (predominantly maghemite) in a multicore structure. This core is coated with a shell, which serves as an anchor layer for a further functionalization of these core/shell particles with the biomolecules. For the anchor layers, two different groups of material are used (see Figure 6.1). First, several types of biopolymers (dextrans with different functional groups) and second, polyelectrolytes and polyampholytes based on polydehydroalanine (PDha) $[60,63,64]$. On the surface of the anchor layer, biomolecules from a natural source (fetal calf serum - FCS) are coupled by an adhesive process.

6.2.1.1 Magnetic cores: The magnetic iron oxide nanoparticles used in this paper were prepared similar to the well-known wet chemical precipitation methods [65] but using another alkaline medium [66, 67]. For this, a $\mathrm{NaHCO}_{3}$ solution was directly added to a $\mathrm{FeCl}_{2} / \mathrm{FeCl}_{3}$ solution and a brownish precipitate of nonmagnetic iron carbonates occurred. After the addition of distilled water, the particles were boiled for $5 \mathrm{~min}$ at $100{ }^{\circ} \mathrm{C}$. In this way, magnetic iron oxides were formed under the release of $\mathrm{CO}_{2}$ and the color 


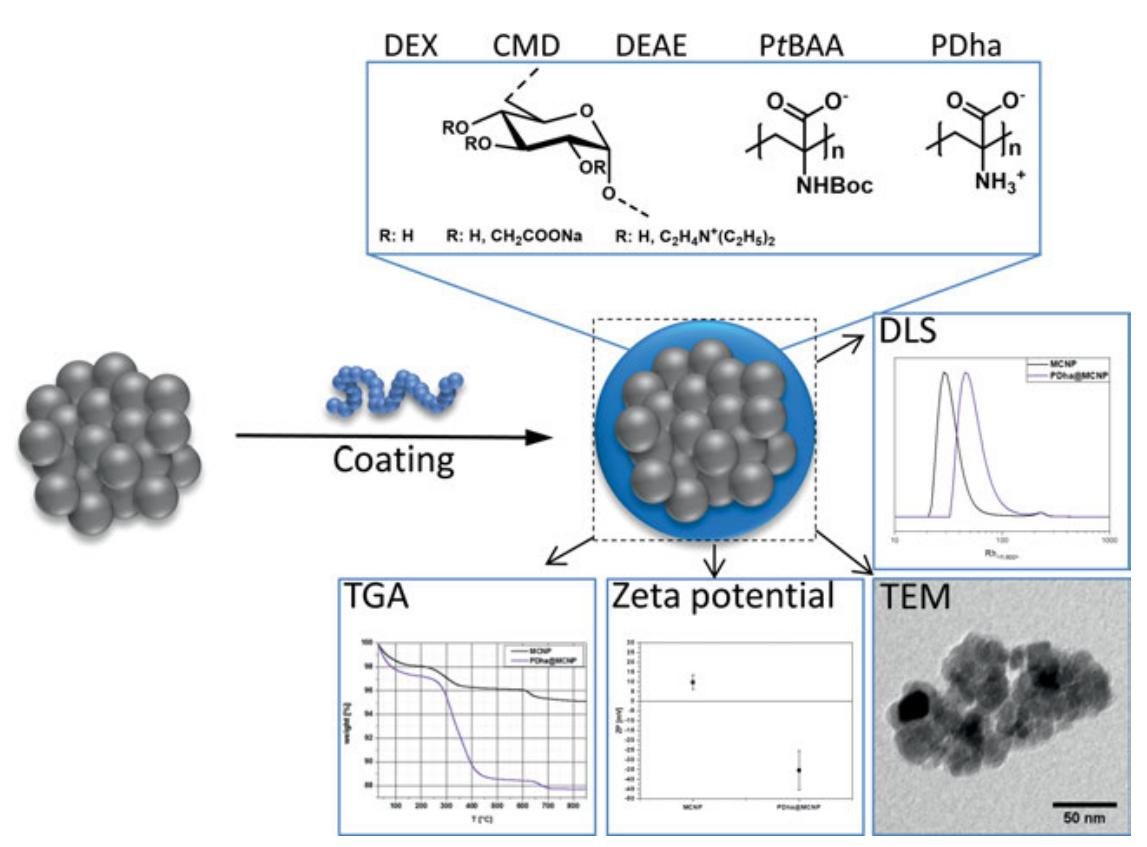

Figure 6.1: Schematic representation of the different core/shell magnetic nanoparticles (MNPs) and the technologies for their characterization.

of the solution turned black. Afterward, the obtained MNP suspension was washed by magnetic separation with distilled water using a high-performance permanent magnet to remove excess educts. The addition rate of the alkaline medium was varied to enable preparation of monodomain single core particles or to prepare clusters of about $50 \mathrm{~nm}$ consisting of several monodomain single cores, showing a superferrimagnetic behavior due to their multicore structure [67-69]. The particles size was controlled by adjusting the temperature and addition rate of the alkaline medium during particle preparation.

\subsubsection{Anchor layer}

6.2.1.2.1 Dextrans: For the investigation of the influence of particle surface charge on the formation of the biomolecule corona, MNP were coated with different materials (dextran - DEX, carboxymethyldextran CMD, diethylaminoethyldextran - DEAE; all purchased from Sigma-Aldrich, Schnelldorf, Germany). These materials have a neutral dextran backbone but different substitution patterns and thus enable a variation of surface charge (DEX - neutral, CMD - negative, DEAE - positive). For coating the MNP with dextran and its derivatives, the nanoparticles were dispersed by ultrasonic treatment (Sonopuls GM200, Bandelin electronic, Berlin, Germany) for $1 \mathrm{~min}$ and sonicated for $1 \mathrm{~min}$ in an ultrasonic bath ( $\mathrm{S} 100 \mathrm{H}$, Elmasonic, Germany). $\mathrm{HCl}$ was added to adjust the $\mathrm{pH}$ value at 2-3 and the suspension was tempered at $45^{\circ} \mathrm{C}$ in a water bath and stirred. At the same time, the coating agents were dissolved in distilled water in a mass ratio (coating/core) of 1:1. The so prepared coating solution was steadily added to the nanoparticle suspension and stirred for $1 \mathrm{~h}$ at $45^{\circ} \mathrm{C}$. Afterward the suspension was treated with ultrasound as described above, washed magnetically twice with distilled water to remove excess coating material, and the desired concentration was adjusted by adding distilled water. 
6.2.1.2.2 Polyelectrolytes and polyampholytes: To study the influence of net charge and charge density, polyampholytic and polyzwitterionic coatings were investigated with regard to the corona formation [55]. Here, the magnetic cores were coated with polyanionic or polyzwitterionic polymeric shells based on PDha. For this, polyanionic poly(tert-butoxycarbonylamino acrylic acid) (PtBAA) and polyzwitterionic PDha were used as obtained by deprotection of poly(tert-butoxycarbonylamino methacrylate) (PtBAMA) as described earlier [64]. The corresponding polyelectrolyte was dissolved in $0.01 \mathrm{M} \mathrm{NaOH}$ and titrated to $\mathrm{pH}=5$ using $0.01 \mathrm{M} \mathrm{HCl}$. The MNP were treated with ultrasound for $10 \mathrm{~min}$ and magnetically separated afterward. Subsequently, the respective MNPs were dispersed in the polyelectrolyte solution at constant weight ratios (MNP:polyelectrolyte $=1: 8)$ and the mixture was ultrasonicated for $1 \mathrm{~h}$, magnetically separated and washed with aqua bidest five times. We further investigated the dependence of shell thickness and stability on different parameters such as solution $\mathrm{pH}$, polymer/MNP ratio, and reaction time or temperature [60].

6.2.1.3 Biomolecule coating: For producing a protein/biomolecule corona around MNP, the particles have to be incubated in a natural protein source, which leads to an accumulation of proteins on the surface of the MNP. For our studies, FCS was used as natural protein source. FCS was obtained from Biochrom GmbH (Berlin), tested for mycoplasma and viruses, tested for endotoxins, and heatinactivated at $56^{\circ} \mathrm{C}$ for $1 \mathrm{~h}$ prior to use.

FCS incubation of MNP was performed by water bath heating resulting in a homogeneous temperature distribution throughout the sample. For water bath incubation, FCS was tempered at defined temperatures in a water bath. A suspension volume containing $15 \mathrm{mg}$ of prepared MNP was added to $2 \mathrm{~mL}$ of tempered FCS and kept at the same temperature for a defined time. Incubation time starts with the addition of the suspension. During the time of incubation ultrasonic treatment at the given temperature was carried out (S100H, Elmasonic, Germany) to redisperse possible agglomerates. After incubation, the suspensions were taken out of the water bath and put on a magnet for magnetic separation, excess FCS was withdrawn and distilled water was added. The washed incubated nanoparticle suspensions were kept at $4{ }^{\circ} \mathrm{C}$ for short-term storage or at $-80^{\circ} \mathrm{C}$ for long time storage [57].

Besides the here described general procedure to prepare a biomolecule/protein corona coating on the surface of MNP, the used incubation parameters were varied in a broad range to study the influence of the incubation procedure on the evolving corona, as described in Section 6.2.2.

6.2.1.4 Size-dependent fractionation: To study the influence of particle size distribution on the stability of the resulting nanohybrid fluids against agglomeration and sedimentation and their interaction with biological systems, size-dependent fractionation experiments by using centrifugation were performed.

In the first approach, the core/shell MNP with the anchor layer only were centrifuged (1-2.5 min at $1000-5000 \times g$ ) to remove agglomerates and obtain narrowly size distributed particle ensembles. Afterward, the obtained samples with narrowed size distribution were biomolecule coated by FCS incubation as described above.

In a second approach, the size-dependent fractionation was performed after the corona formation. For this, the unfractionated core/shell MNPs were incubated in FCS at $37^{\circ} \mathrm{C}$ for $10 \mathrm{~min}$ to obtain FCS precoated MNP. To remove excess FCS and loosely bound biomolecules to obtain MNPs with a so-called "hard corona", incubated core/shell MNPs have been washed magnetically four times with distilled water and were redispersed by vortexing and ultrasonication. Afterward, MNPs were centrifuged (1$3 \mathrm{~min}$ at $1000-5000 \times \mathrm{g}$ ) and the supernatants, containing narrowly size distributed MNPs, were used for further investigation. 


\subsubsection{Control of corona formation}

For the investigation, if the total amount of resulting biomolecule corona on the surface of the MNP can be modified by the incubation medium, FBS (Biochrom, Germany) diluted in cell culture medium (RPMI $1640+$ GlutaMAX $^{\mathrm{TM}}$, Life Technologies, USA) was used for the incubation of commercially polyethylenimine (PEI)-coated MNP (fluidMAG-PEI/B and fluorescence marker-labeled nanoscreenMAG/ G-PEI/750/O, chemicell, Berlin, Germany). Additionally, we used these cell toxic MNP with a PEI coating, to investigate a putative protecting effect of a biomolecule corona with regard to cytotoxicity. For the incubation, a water bath heating at $37^{\circ} \mathrm{C}$ for $10 \mathrm{~min}$ was used. To modulate the amount of proteins bound to MNP the composition of the incubation medium was varied. Starting from pure FCS, the amount of FCS in RPMI 1640 was reduced continuously down to an FCS concentration of $0 \%(\mathrm{v} / \mathrm{v})$. The resulting biomolecule corona was characterized regarding its protein composition and total biomolecule amount on particles surface.

For the investigation of the influence of particle surface charge on the formation of the biomolecule corona, coatings with a neutral dextran backbone but different substitution patterns (DEX, CMD, DEAE) resulting in different surface charge (DEX - neutral, CMD - negative, DEAE - positive) were incubated in FCS and the resulting biomolecule corona was characterized.

During the preparation of the biomolecule corona, the process parameters incubation temperature and time were varied to study their influence on the resulting biomolecule corona. For this, the incubation was performed in a water bath as described above at defined incubation temperatures $(25,37,50$, $70^{\circ} \mathrm{C}$ ). To study the influence of incubation time, the suspensions were taken out of the water bath (at different temperatures) at defined incubation time points (1, 5, 10, $20 \mathrm{~min}$ ) and put on a magnet for magnetic separation. Excess FCS was withdrawn and distilled water was added to terminate the incubation process.

To investigate, if the biomolecule corona formation can be prevented or at least reduced, corona formation on polyzwitterion-coated MNP was performed for the above described parameters and the resulting corona was compared to that one obtained on negatively charged (similar charge as the biomolecules) MNP. For this, polyzwitterionic (PDha) and anionic (PtBAA)-coated MNP were used for incubation and the resulting biomolecule corona was characterized regarding its protein composition and total biomolecule amount on the particle surface.

\subsubsection{Sterilization/preservation}

For a potential application of the biomolecule-coated MNPs in animals or humans, the particles have to be free from biological pathogens or any biological contamination. For particles smaller than $220 \mathrm{~nm}$, filtration can be used for sterilization. Unfortunately, this method is not suitable for sterilization of herein used larger biomolecule-coated particles $(>220 \mathrm{~nm})$ because the membrane cut off for sterile filtration is $220 \mathrm{~nm}$.

Furthermore, a method for preservation of biomolecule-coated MNP is needed, which guarantees storage for several months without an alteration of the integrity of the biomolecule coating. In previous experiments, we found a complete decay of the protein coating after a few days for storing in the fridge $\left(4^{\circ} \mathrm{C}\right)$.

Up to now, standard methods for sterilization and preservation of proteins, which are the main proportion of the biomolecule corona, are UV sterilization and lyophilization, respectively. To our knowledge, such procedures were never tested for sterilization and preservation of biomolecule-coated MNP. Therefore, the aim of our study was to establish procedures, which allow a reliable sterilization and preservation of larger biomolecule-coated MNP without any damaging effect on the integrity of the biomolecule corona. For this, biomolecule-coated DEAE-MNP were prepared as described above, 
treated by different procedures for sterilization and preservation (UV sterilization, autoclaving, freezing, and lyophilization), and characterized afterward regarding possible damaging effects on the integrity of the biomolecule coating as well as other negative effects like agglomeration etc. More details about the used protocols for the treatments are described in Dutz et al. [57].

6.2.3.1 UV sterilization: To sterilize the biomolecule-coated MNPs, the magnetic nanohybrids were exposed to a UV-C radiation with a wavelength in the range from 200 to $280 \mathrm{~nm}$. About $254 \mathrm{~nm}$ is the optimum wavelength to damage nucleic acids and thus the DNA of organisms [46], which kills the potentially existing germs within the sample. The exposure time was adjusted for a first sample set to be $150 \mathrm{~min}$, representing the minimum necessary time for the sterilization as confirmed in a previous study [57], and for a second sample set to be $240 \mathrm{~min}$, representing a treatment time for sterilization including a safety margin.

6.2.3.2 Autoclaving: A standard protocol for autoclaving [70] was used to sterilize the biomoleculecoated MNP. The biomolecule coated MNP suspension was placed into a Varioklav $25 \mathrm{~T}$ (Thermo Fisher, USA) and exposed to a temperature of $121{ }^{\circ} \mathrm{C}$ for $20 \mathrm{~min}$ at a pressure of $3 \mathrm{bar}$. At this increased temperature, the DNA of pathogens in the sample is damaged due to protein denaturation and leads to a die-off of the pathogens.

6.2.3.3 Freezing: The reproduction of bacteria is slowed at low temperatures and reaches a standstill at temperatures below $4^{\circ} \mathrm{C}[71]$. The samples were frozen at $-15^{\circ} \mathrm{C}$ (freezing) and $-80^{\circ} \mathrm{C}$ (deep-freezing) to check the impact of freezing-time on the structure of the nanohybrids. For this, at each time point six samples of $1 \mathrm{~mL}$ of the frozen protein-coated MNP suspension were stored at $-15^{\circ} \mathrm{C}$ and $-80^{\circ} \mathrm{C}$ for up to 6 weeks. At distinct time points (1 day, 2 days, 1 week, 2, 4, 6 weeks) after freezing, the samples were defrosted and characterized immediately.

6.2.3.4 Lyophilization: Lyophilization is a well-established method for preservation of aqueous protein-based pharmaceuticals [72]. During the lyophilization procedure, all the water from the sample is removed, which leads to a complete cessation of the vital function of the pathogens as well as to a complete standstill of their reproduction. Biomolecule-coated MNP samples used in this study were lyophilized, and the dry samples were stored at $4{ }^{\circ} \mathrm{C}$. At distinct time points $(1,3$, and 6 weeks after lyophilization), the dry powder samples were redispersed and characterized regarding the occurrence of larger agglomerates within the suspensions and if the lyophilization procedure influences the integrity of the biomolecule structure. To determine the influence of additives on the redispersion of the dry powder samples, a second set of samples was mixed with tetramethylammonium hydroxide (TMAH) or polyethylene glycol (PEG), which are known as additives to enhance the suspensions stability against agglomeration and sedimentation of ferrofluids, and treated as described above.

\subsubsection{Characterization of the samples}

The magnetic characterization of all types of particles was done by vibrating sample magnetometry (VSM, Micromag 3900, Princeton Measurement Corporation, Princeton, USA) at room temperature. From liquid samples and dry powders, the saturation magnetization (Ms), the coercivity (Hc), and the relative remanence $(\mathrm{Mr} / \mathrm{Ms})$ were determined. Usually, drying of ferrofluids to powders leads to an alteration of the magnetic properties of the single cores due to increasing magnetic interactions between the single cores, when the distance between the cores is reduced [73]. Therefore, the obtained values for $\mathrm{Hc}$ and $\mathrm{Mr} / \mathrm{Ms}$ are interpreted qualitatively only. Since Ms is mainly unaffected from magnetic interactions, this value can be used for quantitative analysis. Taking into account the measured Ms of 
the plain magnetic cores, the particle concentration within the fluidic samples was calculated. From the Ms of the dry samples, the proportion of nonmagnetic material on the surface of the MNP, which can be attributed to the coating material and/or the biomolecule corona, was estimated.

The inner structure of the magnetic MCNP was investigated by means of X-ray diffraction (XRD, Panalytical X'pert Pro, Malvern Panalytical, Almelo, The Netherlands). The results of the XRD investigations gave information about magnetic phase composition [74] and the mean sizes of the primary cores, which form the multicore particle. The size of the primary cores was calculated from measurements of the XRD line width by using the Scherrer formula. The physical cluster size and shape as well as the agglomeration behavior of all different particles was derived from transmission electron microscopy (TEM, Tecnai ${ }^{\text {TM }} \mathrm{G}^{2}$ 20, FEI company, Hillsboro, USA) images. Hydrodynamic diameters (dh) and its polydispersity index, as well as surface charge (zeta potential) of the core/shell MNP and the hybrid nanoparticles were determined by using dynamic light scattering (DLS, Zetasizer nano ZS, Malvern Instruments, Malvern, UK). Before the measurement, samples were diluted in the ratio 1:30 with distilled water and treated in an ultrasonic bath. For size measurements, the $z$-average of the intensity weighted normalization was used. To measure the zeta potential, the medium viscosity and dielectric constant were taken from water at $25^{\circ} \mathrm{C}$ with $0.8872 \mathrm{cP}$ and 78.5 , respectively. All measurements were performed in three consecutive runs and obtained values were averaged.

For the determination of the mass of biomolecules bound to the particles surface, thermogravimetric analysis (TGA, STA409, Netzsch, Selb, Germany) was used. Samples were freeze-dried to obtain fine dry powders for TGA experiments. These samples were heated from room temperature up to $330^{\circ} \mathrm{C}$ and the corresponding mass loss was continuously determined. The obtained curves for biomoleculecoated MNP were normalized to curves for plain MNP and core/shell MNP.

The determination of the composition and the amount of the proteins of the biomolecule corona on the surface of the nanohybrids was performed by means of sodium dodecyl sulfate polyacrylamide gel electrophoresis (SDS-PAGE). For this, $2 \times$ Laemmli sample buffer (Bio-Rad, Munich, Germany) supplemented with 2-mercaptoethanol (final conc. $355 \mathrm{mM}$ ) was added to the samples in the first step and heated up to $95^{\circ} \mathrm{C}$ to destroy both secondary and tertiary structure of proteins. Afterward, the denatured proteins were separated by molecular weight with PAGE on a 4-12\% Bis-Tris gel (Bio-Rad, Munich, Germany). After gel electrophoresis, the proteins were visualized by highly sensitive silver staining (SilverXpress Silver Staining Kit, Invitrogen, Heidelberg, Germany). The gel images were processed by ImageJ (National Institutes of Health, Bethesda, USA) [75]. As references, a molecular weight standard protein collection Kaleidoscope marker (Bio-Rad, Munich, Germany) and untreated FBS were used.

To investigate time dependent processes of corona formation, magnetorelaxometry (MRX) was performed to investigate the Brownian relaxation behavior of the MNP as function of corona thickness. For this, a setup, which utilizes fluxgate sensors (FG-MRX) for measuring the magnetization decay, was used [76].

\subsubsection{Interaction with biological systems}

6.2.5.1 Cell viability studies: Cell viability studies were performed utilizing human brain microvascular endothelial cells (HBMECs). HBMECs were cultivated at $37^{\circ} \mathrm{C}$ and $5 \% \mathrm{CO}_{2}$ in RPMI 1640 + Gluta-

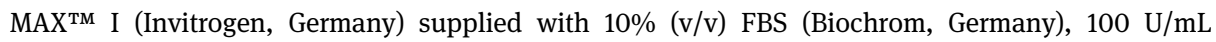
penicillin, and $0.1 \mathrm{mg} / \mathrm{mL}$ streptomycin (Life Technologies, USA). Afterward, the cells were seeded into black-walled 96-well plates ( $\mu$-Clear, F-bottom, Greiner Bio-One, Frickenhausen, Germany) in triplicate and then incubated with particle concentrations between 5 and $100 \mu \mathrm{g} / \mathrm{cm}^{2}$ (corresponding to 19 $378 \mu \mathrm{g} / \mathrm{mL}$ ), water (negative control [NC]), or PEI-coated PEI-M particles (positive control, micromod Partikeltechnologie GmbH, Rostock, Germany). The "PrestoBlue” cell viability Reagent (Thermo Fisher Scientific, Waltham, USA) was added 3 and $24 \mathrm{~h}$ after the seeding and the incubation was continued for 
further $30 \mathrm{~min}$ at $37^{\circ} \mathrm{C}$. After magnetic separation of the MNP to the outer walls of the wells and an excitation of the cells with light of $550 \mathrm{~nm}$ ( $20 \mathrm{~nm}$ bandwidth), a fluorescence signal at $600 \mathrm{~nm}$ (40 nm bandwidth) was detected by using the CLARIOstar microplate reader (BMG LABTECH GmbH, Orthenberg, Germany). By measuring cell free wells with added particles, the particle-associated auto fluorescence effect was measured and used for correction of cell measurements. Similarly, quenching effects were considered by fluorescence measurements immediately before and after addition of particles to the cells in the wells. From these investigations, the cell survival rate (which is an indicator for cell toxicity of the particles) was derived for different particle concentrations for the above mentioned time points of 3 and $24 \mathrm{~h}$ after start of particle-cell incubation.

6.2.5.2 Flow cytometry: Furthermore, the interaction of biomolecule-coated MNP and living HBMECs was investigated by means of flow cytometry based on the cellular loading with fluorochrome-labeled nanoparticles $\left(\lambda_{\mathrm{abs}}=476 \mathrm{~nm}, \lambda_{\mathrm{em}}=490 \mathrm{~nm}\right)$. First, incubated cells were washed and harvested using accutase (PAA Laboratories, Austria). Second, after a centrifugation step $\left(5 \mathrm{~min}, 200 \mathrm{rcf}, 4^{\circ} \mathrm{C}\right)$ the cell pellet was resuspended in $10 \%(\mathrm{w} / \mathrm{v})$ formalin (Sigma-Aldrich, Germany) and fixed at room temperature for $15 \mathrm{~min}$. This washing procedure was performed in triplicate. After that, the cells were analyzed with the FACSCalibur cytometer (Becton Dickinson, USA) with 2500-10,000 cell counts per sample depending on recoverable cells and cytotoxicity of respective particles. By application of the Becton Dickinson's software "CellQuest Pro", the geometric mean value for fluorescence from cells only was determined and correlated to the condition of the cells in dependence of the amount of incubated particles.

6.2.5.3 Confocal laser scanning microscopy: More detailed investigations on cellular particle interaction and uptake were performed by using confocal laser scanning microscopy. For this, HBMECs were seeded on glass cover slips (12 mm diameter, Menzel, Germany) placed within a 24-well plate. The HBMECs were incubated with fluorescence-labeled MNPs as described above. After the incubation process, the cells were washed three times with phosphate buffered saline (Invitrogen, Germany) and fixed in 10\% (v/v) neutral buffered formalin (Sigma-Aldrich, Germany) for $15 \mathrm{~min}$.

This was followed by a permeabilization of the cell membrane in $0.1 \%(\mathrm{v} / \mathrm{v}$ ) Triton X 100 (SigmaAldrich, Germany) for $15 \mathrm{~min}$ and cytoskeletal actin and nuclei were stained simultaneously with Alexa Fluor ${ }^{\circledR} 647$ Phalloidin and Hoechst33258 (both Invitrogen, Germany) for 60 min at $37^{\circ} \mathrm{C}$. The fluorescence from the stained structures was analyzed by using a confocal laser scanning microscope LSM 510 META (Carl Zeiss Microscopy GmbH, Germany) with 400-fold magnification. For this, the samples were scanned with a slice thickness of $1 \mu$ m layer by layer, resulting in $z$-stacked images of $10-15$ slices. As a result, the spatial distribution of the MNP within the cell layer is obtained. A quantification of internalized MNPs was performed by overlapping signals derived from the cytoskeletal actin and the MNP channels by using MATLAB (MathWorks, USA) to analyze the single image stacks.

6.2.5.4 Shell-less hen's egg model: Core/shell MNP and biomolecule-coated MNP were tested for their biocompatibility after systemic injections using a shell-less hen's egg model (see Figure 6.2) according to Schlenk et al. [77]. Briefly, fertilized chicken eggs were obtained from a local supplier and incubated for $72 \mathrm{~h}$. The contents of the fertilized chicken eggs were transferred into petri dishes (Greiner Bio-One $\mathrm{GmbH}$, Frickenhausen, Germany) containing Ringer's solution $\mathrm{pH} 7.0$ to obtain the vascularized CAV at stages 14-17 according to Hamburger and Hamilton [78]. After further $24 \mathrm{~h}$ incubation, MNP samples were injected $(2 \mu \mathrm{L})$ into the vitelline vein using a microinjector (Sutter Instrument, Novato, USA) connected to a borosilicate glass capillary. Directly before injections, selected MNP and biomoleculecoated MNP dispersions were diluted with sterile water to the final test concentrations of 160,500 , or $1000 \mu \mathrm{g} / \mathrm{mL}$. Different fractionated and nonfractionated samples were tested. Branched PEI (25 kDa bPEI, $25 \mathrm{mg} / \mathrm{mL}$ dissolved in sterile water, kindly provided by BASF, Ludwigshafen, Germany) was 


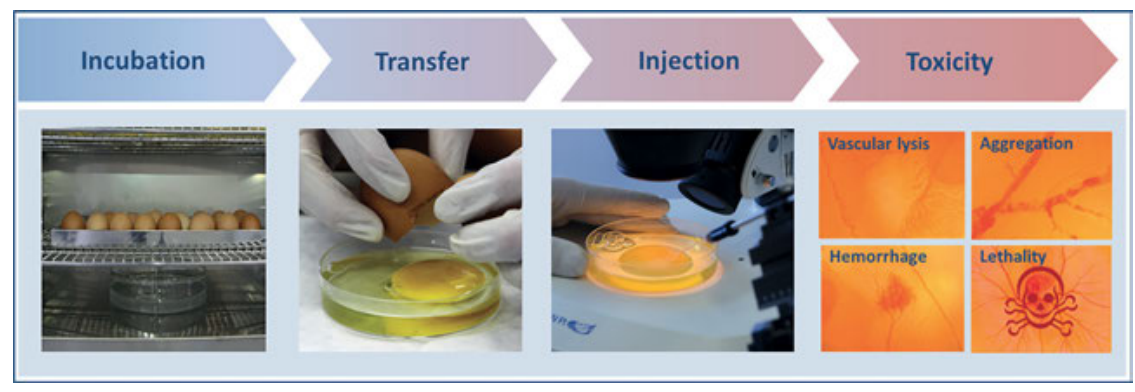

Figure 6.2: Schematic representation of the single work stages during the investigation of in vivo/ex ovo toxicity by means of the shell-less hen's egg model.

injected as positive control. According to the recommendations of the Interagency Coordinating Committee on the Validation of Alternative Method (ICCVAM) for the HET-CAM test [79], 0.9\% $\mathrm{NaCl}$ and sterile water were selected as NC and solvent control, respectively. Eggs were inspected for toxic (hemorrhage, vascular lysis, aggregation) or lethal (stop of the heartbeat) reactions by macroscopic and microscopic observations at different time points $(0,1,2,4,8$ and $24 \mathrm{~h})$ after injection. Historical lab control values were used to confirm the validity of the test procedure. Each sample was tested in two independent experiments with a total number of at least nine eggs.

\subsection{Results and discussion}

\subsubsection{Properties of magnetic cores/shell particles}

Magnetic nanoparticle cores were prepared from a wet chemical precipitation process. By variation of reaction temperature and velocity, single core and multicore structures were obtained.

Figure 6.3A shows a typical TEM image of an ensemble of single core MNP. For this type of MNP, the core diameter was determined by means of XRD and TEM and shows a typical value of about $10 \mathrm{~nm}$. The analysis of the 440-peak in the diffractogram confirmed a spinel structure of the lattice with a dominating maghemite $\left(y-\mathrm{Fe}_{2} \mathrm{O}_{3}\right)$ phase and small proportion of magnetite $\left(\mathrm{Fe}_{3} \mathrm{O}_{4}\right)$. The freeze-dried sample shows in VSM measurements at room temperature a saturation magnetization of about $68 \mathrm{Am}^{2}$ / $\mathrm{g}$, a coercivity of less than $0.2 \mathrm{kA} / \mathrm{m}$, and a relative remanence of about 0.005 . The obtained values are in the typical range for maghemite nanoparticles of this size and confirm a superparamagnetic behavior. A size derived from the magnetic properties by applying the Chantrell method [80] revealed a value of $9.6 \mathrm{~nm}$, which is in good accordance to size from XRD and TEM, which is a further evidence of superparamagnetic behavior.

For the multicore MNPs, a cluster size of about $50 \mathrm{~nm}$ was measured by TEM (see Figure 6.3B) and the primary core size of the single cores forming the cluster was $11 \mathrm{~nm}$, 

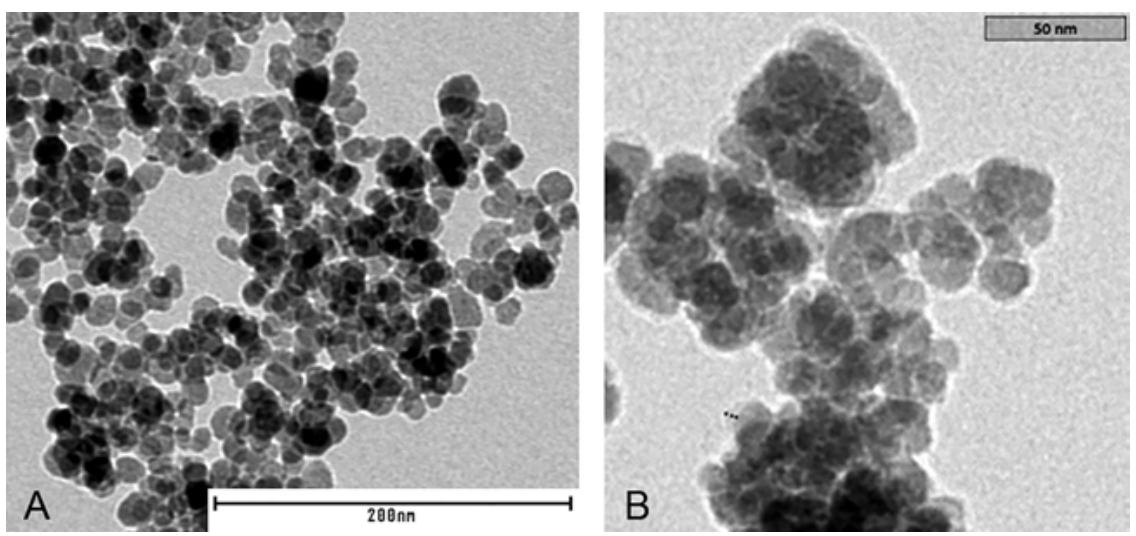

Figure 6.3: Typical transmission electron microscopy (TEM) image of the prepared magnetic nanoparticles; (A) pristine single core magnetic nanoparticles (MNPs) and (B) multicore MNP coated with polydehydroalanine (PDha).

measured by means of XRD. From these findings, it was confirmed by TEM that the multicore structure exists already for the pristine particles and is not an effect of the coating process. Analysis of the 440-peak in XRD revealed that the magnetic phase composition of the MCNP is slightly shifted towards magnetite, compared to the phase of the single cores. Dry powders of the plain MCNP show a Ms of about $74 \mathrm{Am}^{2} / \mathrm{kg}$, a coercivity of about $2.6 \mathrm{kA} / \mathrm{m}$, and a relative remanence of about 0.06 . These measurements confirm a ferrimagnetic behavior of the MCNP in the presence of a magnetic field.

After coating the magnetic cores (single cores as well as multicores) with different dextrans (Dex, CMD, DEAE) and dispersing the core/shell particles in water, sedimentation stable ferrofluids were obtained. Depending on the coating, the zeta potential of the MNP $(+35 \mathrm{mV})$ was shifted to $+60 \mathrm{mV}$ for DEAE, $+2 \mathrm{mV}$ for Dex, and $-33 \mathrm{mV}$ for CMD. For all different types of coating, a variance of the zeta potential in the range of $\pm 5 \mathrm{mV}$ was observed, which results from the accuracy of the measurement technology.

For the coating of the MNP with PDha and PtBAA, it could be shown using a combination of TEM, DLS, and TGA that well-defined polymeric shells of a few nanometer thickness could be formed [58]. The net surface charge was determined for both coatings to be about $-40 \mathrm{mV}$. The surface charge pattern is polyanionic for PtBAA and polyzwitterionic for PDha-coated MNP. In addition, it turned out that MNP@PDha core-shell hybrid nanoparticles exhibit similar charge characteristics as the shell material (PDha) itself, allowing to invert the surface charge of the hybrid particles [61]. This could later on be used to demonstrate reversible adsorption/desorption of either polyelectrolytes, small charged molecules as model for the removal of pollutants from aqueous media, or even BSA as model protein [81]. 


\subsubsection{Formation of the biomolecule corona}

For producing a protein/biomolecule corona around MNP, the particles were incubated in a natural protein source (FCS), which leads to an accumulation of proteins on the surface of the MNP. In this section, we discuss the general formation process of a biomolecule corona around MNP. The influence of the surface of the different core/ shell MNP and the incubation parameters on the evolving corona are discussed in Section 6.3.3.

Figure 6.4 shows the zeta potential of pristine uncoated single core MNP, as well as for MNP with DEAE, DEX, and CMD coating prior and after incubation in FCS for $10 \mathrm{~min}$ at $25^{\circ} \mathrm{C}$. It is clearly to see that the serum incubation changes the zeta potential of the samples significantly. For all samples, the surface charge turns to negative $(-32$ to $-41 \mathrm{mV}$ ) after serum incubation. Because proteins and their amino acids, as well as other biomolecules like lipids have a negative charge at $\mathrm{pH}$ of 7 , these results confirm the formation of a biomolecule corona on the surface of all tested particles. Furthermore, it can be recognized that, a weaker negative/stronger positive surface charge leads to stronger values of the negative surface charge after incubation. This might be explained by a binding of more negative proteins to positive surfaces. This effect is discussed in more detail in Section 6.3.3.

To obtain a general information about the amount of biomolecules bound to the surface of the MNP, freeze-dried powders of pristine MNP incubated in FCS were investigated by means of VSM and TGA (see Figure 6.5). From the VSM measurements (see Figure 6.5A), it can be seen that the Ms of the samples decreases for incubated particles. This effect can be explained by a proportion of nonmagnetic material within the incubated sample, which might be attributed to (nonmagnetic) biomolecules and

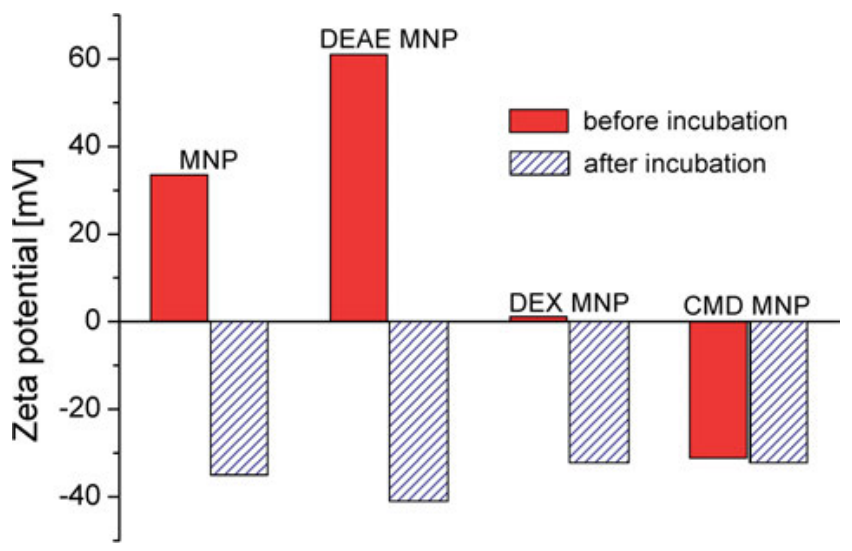

Figure 6.4: Zeta potential of magnetic nanoparticles (MNPs) and for MNPs coated with diethylaminoethyldextran (DEAE)-dextran, dextran, and CM-dextran before (red columns) and after serum incubation (blue hatched columns) confirm formation of a biomolecule corona around magnetic nanoparticles during serum incubation [62]. 
A

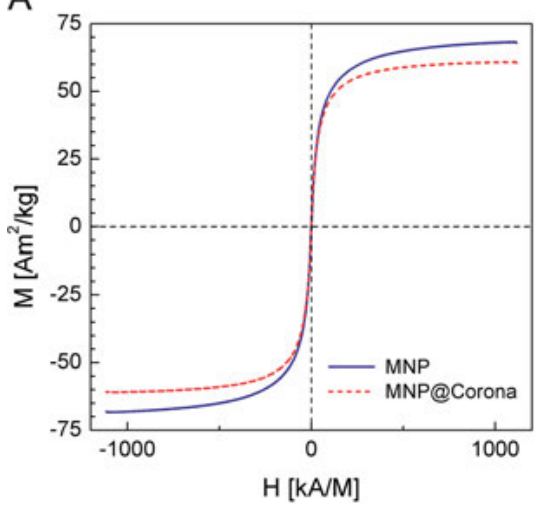

B

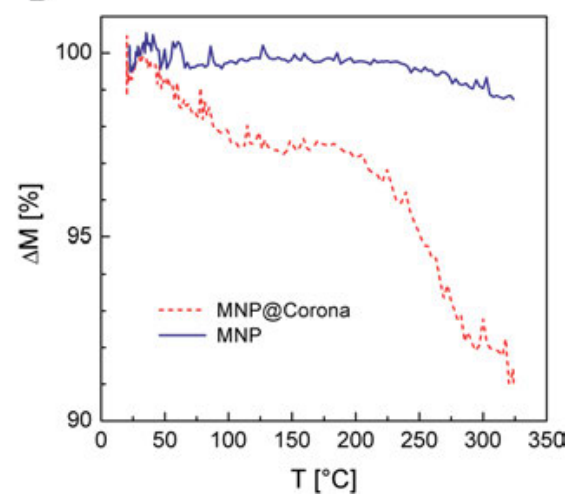

Figure 6.5: (A) Hysteresis curves and (B) TGA investigations of dried pristine, as well as biomoleculecoated magnetic nanoparticles (MNPs) confirm a proportion of biomolecules of about $10 \%$ by mass for the magnetic nanohybrids [62].

confirms the formation of a biomolecule corona on the surface of the MNP. Taking into account a Ms of $68.2 \mathrm{Am}^{2} / \mathrm{kg}$ for the pristine MNP, this decreased to $60.8 \mathrm{Am}^{2} / \mathrm{kg}$ for the incubated particles, the proportion of biomolecules on the particles was estimated to be $10 \%$ by mass.

This value was confirmed by TGA. For this, the same sample (before and after incubation) was heated up to $330^{\circ} \mathrm{C}$ and the mass loss was recorded. For the pristine MNP, a mass loss of about $1 \%$ was found, which is attributed to the evaporation of adsorbed water and crystal water. Investigation of incubated MNPs (see Figure 6.5B) revealed a mass loss of about $9 \%$ in two steps. First, the water evaporates and then the biomolecules on the surface decompose. Comparison of both curves leads to a mean proportion of biomolecules on the nanohybrids of about $8 \%$ by mass. A possible explanation of the lower mass loss in TGA compared to VSM measurements is the fact that heating up to $330{ }^{\circ} \mathrm{C}$ might lead to an incomplete decomposition of the biomolecules.

In a further study, the temporal effects of the biomolecule corona formation were investigated. Because the binding of biomolecules to the surface of the MNP leads to significant changes in relaxation time, the kinetics of corona formation were investigated by means of Flugate-MRX [76]. For this, FCS was added to a core/shell MNP ferrofluid at room temperature and MRX measurement started immediately after the mixing process. Core/shell MNP with DEAE coating was used since these particles seem to have a strong affinity to bind biomolecules (see Figure 6.4). The core size was adjusted to be multicore with a cluster size of $50 \mathrm{~nm}$, since such particles show a blocked magnetism at room temperature, which is a prerequisite to perform MRX measurements.

In the relaxation curves for the investigations on the short-term scale up to $5 \mathrm{~min}$ after mixing (see Figure 6.6A), it can be seen that already for the first measurement $15 \mathrm{~s}$ 
after mixing, a significant change in the relaxation curve compared to the relaxation curve of the nonincubated core/shell MNP occurs. An increase of the relaxation time was observed, which can be attributed to an increase of the hydrodynamic diameter, caused by the formation of a biomolecule corona on the surface of the MNP or an agglomeration of the particles. No significant deviations from this relaxation behavior to the curves for the following $5 \mathrm{~min}$ after mixing were found. This means that the size of the corona remains constant within the investigated time range of $5 \mathrm{~min}$. This was also confirmed by quantitative SDS-PAGE, where a relatively constant amount of bound biomolecules during the first minutes of incubation was observed (data not shown).

On a longer time scale (up to 4 days after mixing), a further decrease of relaxation time was observed (see Figure 6.6B). The most probable explanation for this behavior might be a cross-linking of the surface proteins, leading to the formation of agglomerates as described before [82].

From these experiments, it can be seen that the formation of the biomolecule corona occurs within seconds, and no significant changes takes place within the following minutes. This observed temporal stability is true for the size of the biomolecule corona only, since from these experiments no information about the change of the corona composition as function of time can be derived. The proportion of biomolecules on the magnetic nanohybrids is in the order of $10 \%$ by mass.

\subsubsection{Corona formation and manipulation}

For the investigation, if the total amount of resulting biomolecule corona on the surface of the MNP can be controlled by the incubation medium, FCS diluted in cell culture medium (RPMI) was used for the incubation of the core/shell MNP. To modulate the
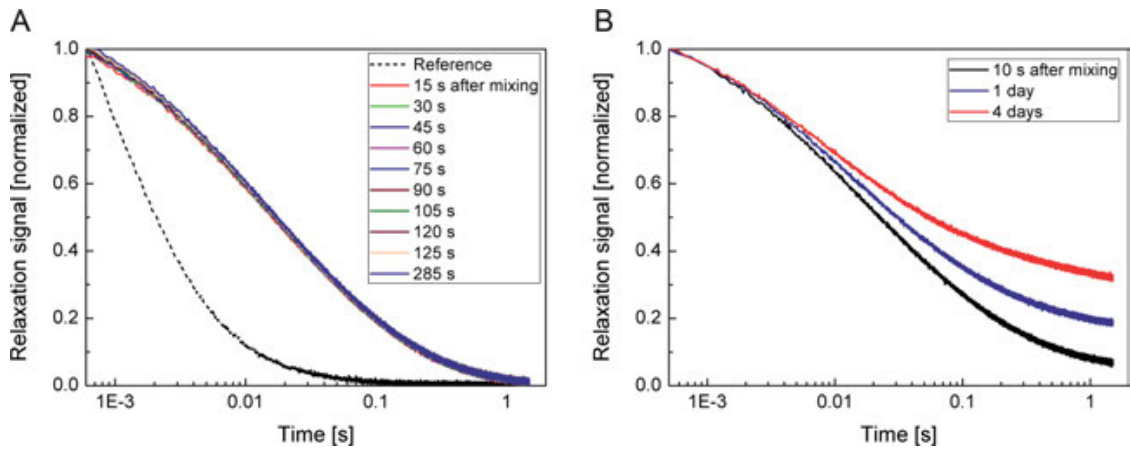

Figure 6.6: Temporal evolution of the magnetorelaxometry (MRX) signal after adding $140 \mu \mathrm{L}$ fetal calf serum (FCS) to $10 \mu \mathrm{L}$ diethylaminoethyldextran (DEAE)-coated magnetic nanoparticles (MNPs) suspension (A) for the first $285 \mathrm{~s}$ after mixing and (B) for incubation times up to 4 days, curves are normalized to be " 1 " for time point $1 \mathrm{~ms}$. For comparison, MRX signal measured on refence sample (10 $\mu \mathrm{L}$ DEAE-coated MNPs suspension diluted with $140 \mu \mathrm{L} \mathrm{DI} \mathrm{H}_{2} \mathrm{O}$ ) is shown in (A) [62]. 
amount of proteins bound to MNP, the composition of the incubation medium was varied from pure FCS down to an FCS concentration of $0 \%$.

For an increasing concentration of FCS in the incubation medium, an alteration of the surface charge of the incubated particles was confirmed. Starting with a zeta potential of $+48.4 \pm 7.8 \mathrm{mV}$ (three independent experiments) for the nonincubated particles, the zeta potential decreased down to zero and turned to negative values for higher FCS concentrations and pure FCS (see Figure 6.7). From this behavior, it can be concluded that a higher FCS amount in the incubation medium leads to the formation of a thicker biomolecule corona. As discussed in Section 6.3.2, the biomolecules are negatively charged at $\mathrm{pH} 7$ and thus, an accumulation of biomolecules on the surface of the MNP leads to changes in the net surface charge depending on bound biomolecule amount. Corresponding to the measured values, a saturation of zeta potential was observed for FCS concentrations above 75 vol- $\%$. A possible explanation for this behavior might be that above a certain FCS concentration, the resulting corona leads to a complete covering of the surface of the particles, and a further increase of FCS concentration does not cause a further growth of the biomolecule corona.

To obtain more detailed information on the amount of biomolecules on the surface and additionally, if the composition of the incubation medium influences the composition of the resulting protein corona too, samples were studied by means of SDS-PAGE. From visual investigation of the gels (see Figure 6.8A), it becomes obvious that a higher amount of FCS in the incubation medium leads to a more pronounced biomolecule corona. ImageJ analysis of the optical density of the protein bands (see Figure 6.8B) reveals again a saturation effect of bound biomolecules on the particles for

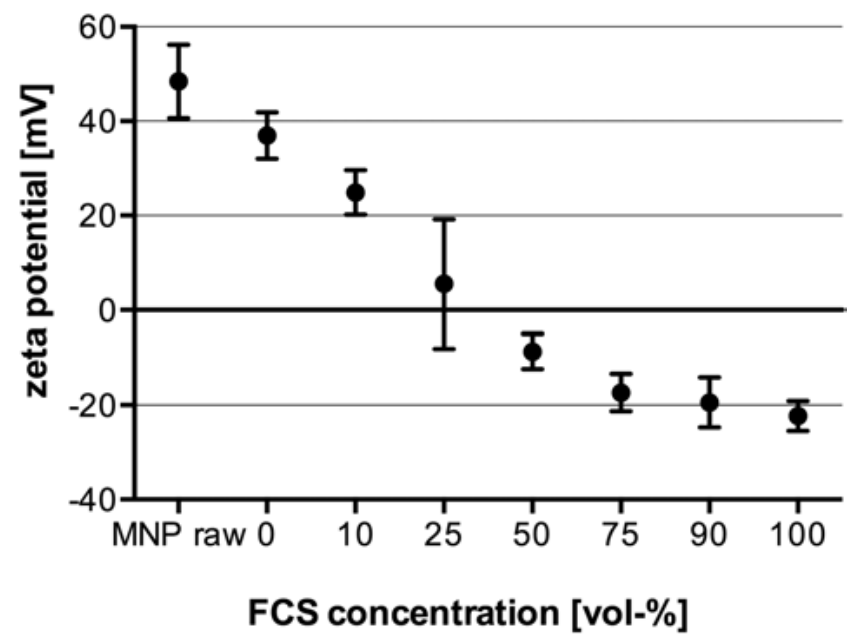

Figure 6.7: Zeta potential of polyethylenimine-magnetic nanoparticles (PEI-MNPs) incubated in RPMI 1640 cell medium containing different amount of fetal bovine serum (FCS) as indicated in a study by Grafe et al. [50]. 

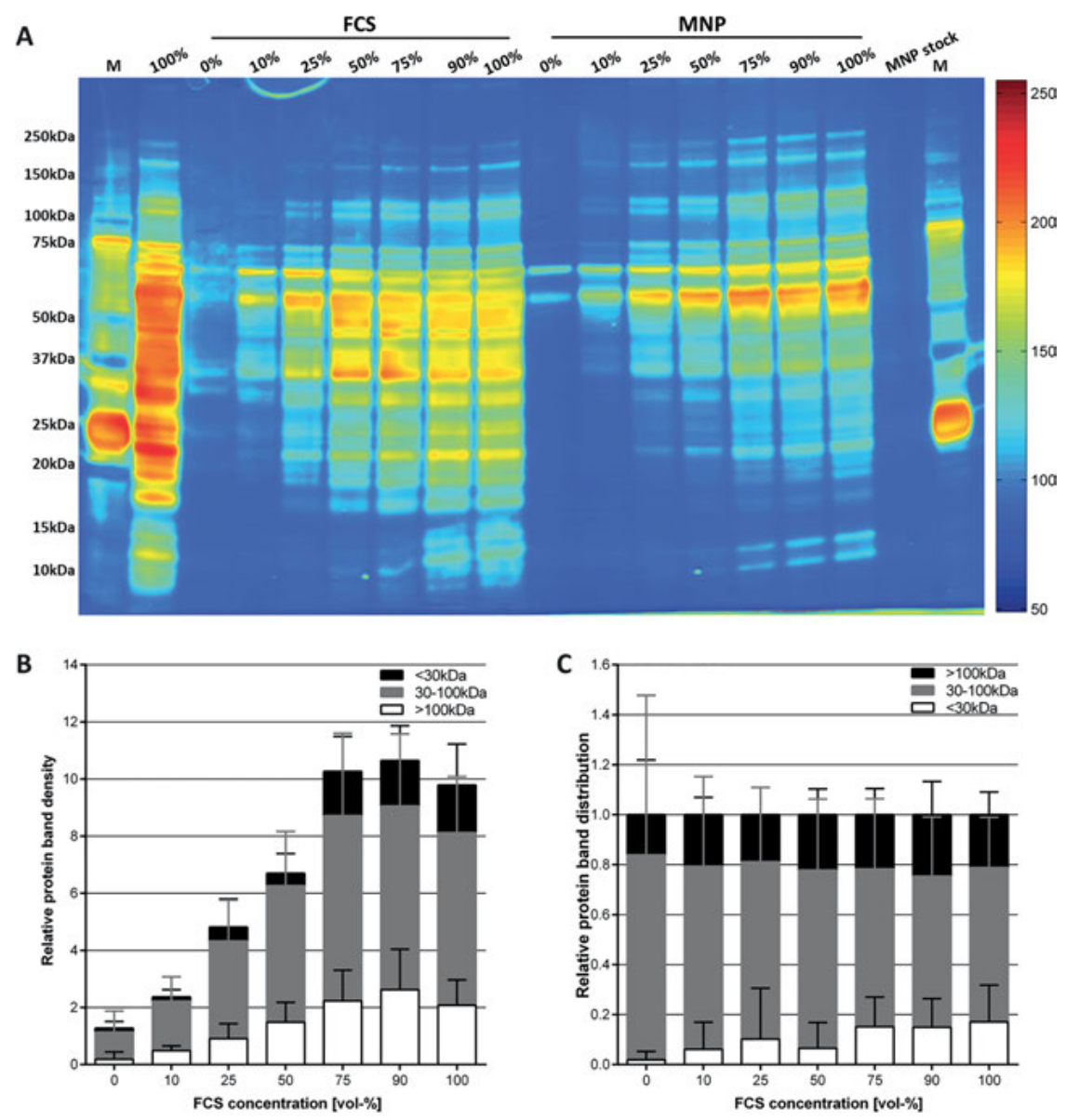

Figure 6.8: Concentration-dependent effect of fetal calf serum (FCS) on biomolecule corona formation on magnetic nanoparticles (MNPs). MNPs incubated with indicated concentrations of FCS (\% [v/v]) were subjected to sodium dodecyl sulfate polyacrylamide gel electrophoresis (SDS-PAGE). Corona-derived proteins were visualized via highly sensitive silver staining. Lanes 10-16 comprise protein bands originated from MNPs, lanes 3-9 show protein bands of respective FCS solution prior to MNP incubation. (A) For better visualization, images are presented in alternative color mapping with low densities shown in blue and high densities in red. (B) Additionally optical density measurements of protein bands of low ( $<30 \mathrm{kDa}$ ), medium (30-100 kDa), and high ( $>100 \mathrm{kDa}$ ) molecular weight are shown relative to $0 \%(\mathrm{v} / \mathrm{v}) \mathrm{FCS}$-treated lanes and (C) their distribution within the respective lanes (C). $M$ is a protein standard marker [50].

FCS concentrations of $75 \mathrm{vol}-\%$ and above, which is in good agreement with the zeta potential measurements.

In general, the protein profiles on the surface of the particles show a similar molecular weight distribution compared to the protein bands for initial FCS used for 
incubation. A quantitative analysis of the relative distribution of three specified protein size classes within each lane by means of ImageJ revealed that the medium-sized proteins (30-100 $\mathrm{kDa}$ ) are dominating (see Figure 6.8C). When the MNP were incubated in media with higher FCS concentrations (75-100\% FCS), the fraction of lowmolecular-weight corona proteins $(<30 \mathrm{kDa})$ increased compared to media with FCS concentrations between 0 and $50 \%$ at the expense of the medium-sized proteins (30$100 \mathrm{kDa}$ ). No influence of the composition of the incubation medium on the amount of large-sized proteins within the biomolecule corona was found and the ratio of highmolecular-weight proteins $(>100 \mathrm{kDa}$ ) remains constant compared to the total corona protein content independent of the applied FCS concentrations.

Resulting from these investigations it can be confirmed that the amount of formed biomolecule corona can be tuned by the content of FCS within the incubation medium. Above a certain concentration of FCS within the incubation medium (here 75 vol-\%), the resulting corona leads to a complete coverage of the particle surface and a further increase of FCS concentration does not cause a further growth of the biomolecule corona. Furthermore, it is possible to alter the resulting composition of the protein corona by variation of the FCS concentration of the incubation medium.

To investigate the influence of incubation temperature and incubation time, both parameters were varied during FCS incubation of pristine MNP as well as PtBAA- and PDha-coated MNP. Figure 6.9 shows the results of SDS-PAGE and TGA investigation of the incubated anionic PtBAA MNP. For all temperatures, the total amount of bound proteins increases with escalating incubation time. Similar results were obtained for pristine MNP [58]. When comparing different incubation temperatures for analogous incubation times, a higher amount of proteins is observed for higher temperatures. Protein size distribution analysis showed that for temperatures up to $50^{\circ} \mathrm{C}$ mostly lowmolecular-weight proteins are bound. At the same time, the amount of medium and high-molecular-weight proteins decreases. Reverse effects were observed for $70{ }^{\circ} \mathrm{C}$, where the fraction of low-molecular-weight proteins decreases with increasing incubation time, and the fraction of medium- and high-molecular-weight proteins increases at the same time. For the incubated pristine MNP very similar results were observed.

TGA investigations for MNP incubated at $25^{\circ} \mathrm{C}$ and $37{ }^{\circ} \mathrm{C}$ showed no remarkable increase in the amount of bound proteins for different incubation times. For temperatures of 50 and $70{ }^{\circ} \mathrm{C}$, an increasing incubation time leads to a higher amount of absorbed proteins. Very similar tendencies were found for incubated pristine MNP.

A different behavior was found for incubated PDha-coated MNP. From SDS-PAGE, it was found that, for all temperatures except $50{ }^{\circ} \mathrm{C}$ a higher protein amount on the surface results for longer incubation times. This effect is very strong at $70^{\circ} \mathrm{C}$. For $50{ }^{\circ} \mathrm{C}$, no influence of the incubation time on the amount of adsorbed proteins was found [58].

To investigate, if the formation of a biomolecule corona can be prevented or at least reduced, FCS incubation of polyzwitterion-coated (PDha) MNP was performed for selected incubation temperatures and durations and the resulting corona was 
compared to that one obtained for same incubation parameters on negatively charged (PtBAA) and uncoated MNP.

From SDS-PAGE, it was found that the highest amount of biomolecules was adsorbed to uncoated (cationic) MNP, followed by (anionic) PtBAA-coated MNP (see Figure 6.10A, B). For the (polyzwitterion) PDha, a significantly reduced protein amount on the surface of the MNP was determined. For all studied samples the majority of the corona consists of medium molar weight proteins (see Figure 6.10C). For PDha instead the proportion of medium molar weight proteins increases with increasing temperatures and incubation times. Also for PDha, the smallest proportion of low-molecularweight proteins was observed. These results were confirmed by TGA (see Figure 6.10D). Again, the highest amount of biomolecules was found for uncoated MNP (7-12\%), followed by PtBAA (5-9\%), and significantly lower for PDha (0.5-2.5\%).

Concluding from these results it can be stated that a clear influence of incubation time and temperature on absorbed protein amount during incubation exists. Usually, longer incubation time and/or higher incubation temperature results in a larger amount of proteins on the surface of the MNP. Additionally, also an influence of surface charge or charge patterns on the protein amount exists. Whilst positive and negative coatings result in very similar results regarding bound proteins on the surface, for
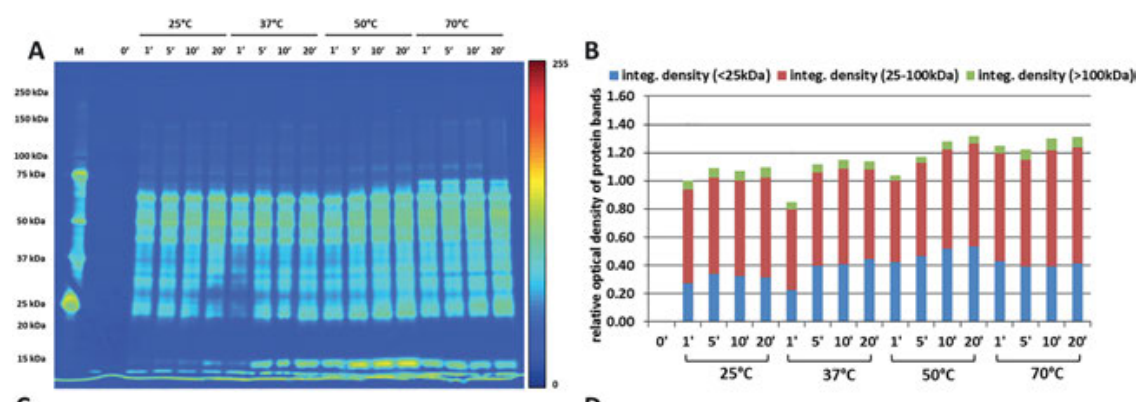

C
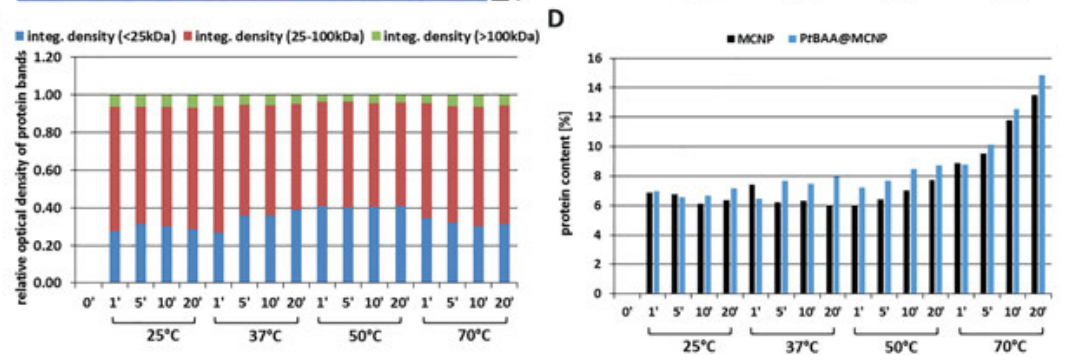

Figure 6.9: Determination of protein content and protein composition of PtBAA-multicore nanoparticles (MCNPs) incubated at different temperatures and times: (A) pseudo color image of protein bands upon sodium dodecyl sulfate polyacrylamide gel electrophoresis (SDS-PAGE) and silver staining, $(B-C)$ relative optical densities of protein bands abstracted from (A), and (D) protein contents calculated from TGA measurements, where the black bars correspond to the pristine MCNPs and the blue bars refer to PtBAA-MCNPs [58]. 


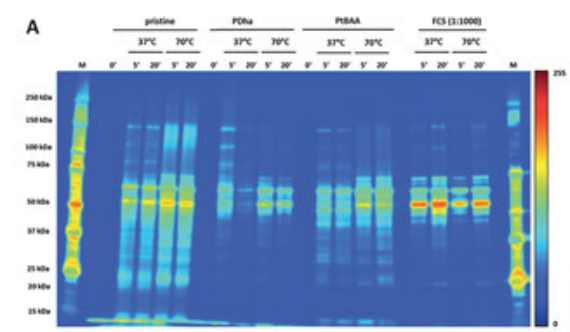

B
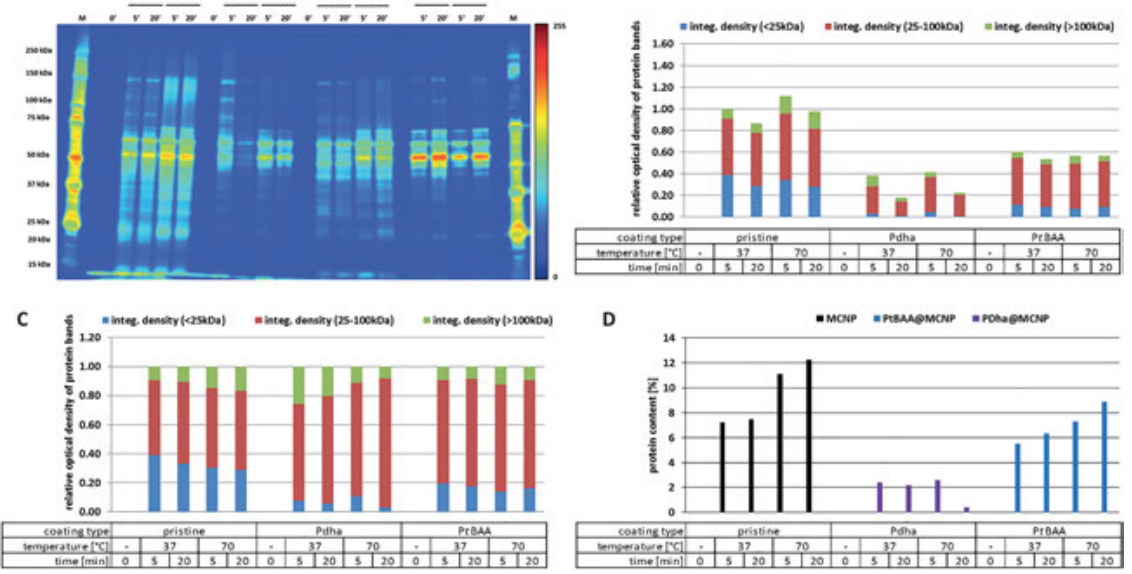

Figure 6.10: Comparison of protein content and protein composition of pristine multicore nanoparticles (MCNPs), polydehydroalanine (PDha)-MCNPs and PtBAA-MCNPs incubated at selected temperatures and for different incubation times: $(A)$ pseudo color image of protein bands upon sodium dodecyl sulfate polyacrylamide gel electrophoresis (SDS-PAGE) and silver staining, (B-C) relative optical densities of protein bands abstracted from (A), and (D) protein contents calculated from TGA measurements [58].

zwitterionic coatings a significantly lower amount of protein was adsorbed to the surface. This might be interesting for the preparation of stealth coatings. As well as for the amount of protein also an influence of the discussed parameters on the composition of the corona was confirmed. But this correlation is too complex to allow description by a simple model and needs support by additional composition analysis to support our findings.

\subsubsection{Sterilization/preservation}

\subsubsection{UV sterilization}

After performing the UV sterilization procedure, samples with 150 and $240 \mathrm{~min}$ exposure time showed no considerable changes in the mean hydrodynamic particles size (see Figure 6.11A), particle size distribution, as well as zeta potential due to exposure to UV radiation. Samples before and after UV sterilization show a zeta potential of about $-30 \mathrm{mV}$, which confirms the presence of a biomolecule corona and that there is no influence of UV exposure on the biomolecule amount on the surface of the magnetic nanohybrids. 
SDS-PAGE revealed no evidence for a UV radiation caused change of the protein integrity. The pattern of the pseudo color greyscale image (see Figure 6.11B) of the original sample (UV-0) looks very similar to the samples exposed to UV radiation for $150 \mathrm{~min}$ (UV-150) and for $240 \mathrm{~min}$ (UV-240). ImageJ analysis of the PAA gel (see Figure 6.11C) shows that in the frame of the measurement accuracy the corona proteins for the different samples have an identic size distribution.

From these measurements we conclude that exposure to UV radiation for up to 240 min causes no relevant changes in the protein amount and integrity und thus, UV sterilization is suitable for the application to biomolecule-coated MNP.

\subsubsection{Autoclaving}

After autoclaving of the biomolecule-coated MNP, a significant decrease of the hydrodynamic particles size and particle size distribution was observed. A higher density of the surface proteins results due to coagulation of the proteins during the autoclaving at elevated temperatures up to $121^{\circ} \mathrm{C}$ could be a probable explanation. This leads to a

(a)
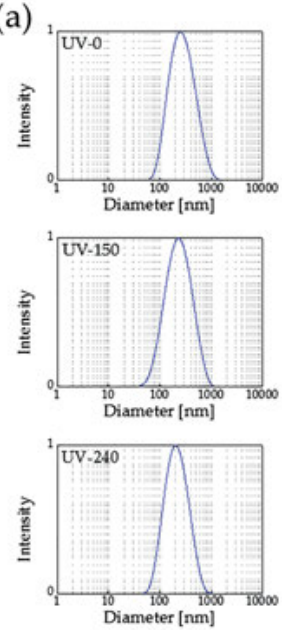

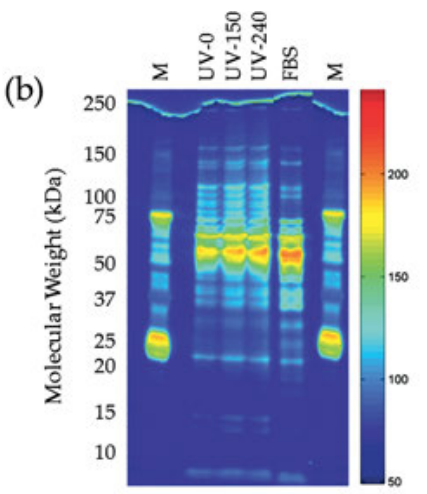

(c)

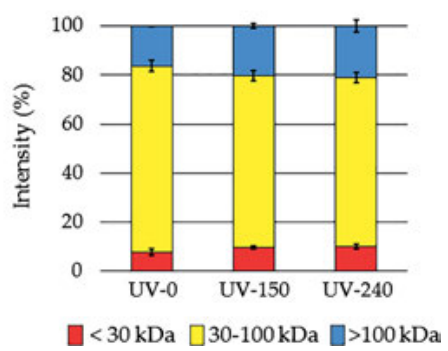

Figure 6.11: Ultraviolet (UV) sterilization of biomolecule-coated nanoparticles does not affect the protein corona. (A) DLS measurements show that the hydrodynamic diameter of the protein-coated nanoparticles decreases slightly with increasing treatment time. (B) Protein size distribution is not altered as demonstrated by gel electrophoresis (silver staining and false color representation). (C) Variations of the protein size ranges over the storage period of 6 weeks [57]. 
lower volume of the biomolecule coating on the core/shell MNP and thus a smaller size is measured. SDS-PAGE investigations revealed strong damages in the protein structure. In the PAA gel, the protein bands are no longer separable but appear as a smear, especially for the high-molecular-weight proteins. All of the typical peaks are vanished after autoclaving, which is attributed to a strong coagulation, denaturation, and degradation of the proteins [57].

From the obtained results, we conclude that autoclaving is not suitable to sterilize biomolecule-coated MNP.

\subsubsection{Freezing}

The visual investigation of thawed samples showed large agglomerates and significant changes in the rheology of the fluids for storing times of two weeks or longer at -15 and $-80^{\circ} \mathrm{C}$. This was confirmed by DLS where agglomerates up to $1000 \mathrm{~nm}$ were found for samples stored for 6 weeks [57]. Despite the zeta potential provided a constant value of about $-30 \mathrm{mV}$ for all samples, it became obvious from the size measurements that during the storage at -15 and $-80{ }^{\circ} \mathrm{C}$ a significant change of the integrity of the biomolecule coating takes place. This was confirmed by SDS-PAGE. For longer storage times, a decrease of the high-molecular weight proteins was observed combined with an increased proportion of the low-molecular weight proteins. This behavior can be interpreted as a degradation of the larger proteins to smaller fragments.

In summary, freezing and storing biomolecule-coated $\mathrm{MNP}$ at -15 and $-80^{\circ} \mathrm{C}$ is not suitable for long-term storage of such magnetic nanohybrids. Short-term storage of up to one week seems to be possible.

\subsubsection{Lyophilization}

The biomolecule-coated MNP samples were freeze-dried in the condition as prepared (plain) and after addition of PEG or TMAH.

PEG was used because it is suggested as cryoprotectant in combination with sugars, and the inherent sugars in serum were expected to be sufficient for this approach.

Similar behavior in particle size and agglomeration was found in each single sample series (plain, PEG, TMAH) for all three different re-dispersion time points (see Figure 6.12A). After redispersion the PEG samples show a similar size and size distribution like the original sample before drying. The plain samples show a slightly higher mean hydrodynamic diameter caused by a weak proportion of not redispersed agglomerates. For TMAH sample, a large proportion of agglomerates remains after redispersion, and the fluid is not stable against sedimentation. The zeta potential showed a constant value of about $-30 \mathrm{mV}$ for the original and all Plain and PEG samples and the zeta potential of all TMAH samples was in the range of -42 to $-44 \mathrm{mV}$. From these observations, a change of the biomolecule integrity due to the addition of TMAH can be expected. 
No remarkable changes in the integrity of the biomolecule coating can be observed in SDS-PAGE for plain and PEG sample for a storage time of one week (see Figure 6.12B). TMAH samples show an alteration of the protein integrity already after one week storage. After 3 weeks storage, the sample plain and PEG exhibit an increase in the lowmolecular-weight fraction, but the protein distribution remains unchanged. After 6 weeks storage, a very slight degradation of the larger proteins occurs and the fraction of small protein fragments increases (see Figure 6.12C).

In summary, the lyophilization of biomolecule-coated MNP after the addition of PEG might be a suitable method for the preservation of such particles for some weeks. The redispersed particles show similar size and stability like the original sample. For storage times longer than 1 week, a partial degradation of the corona proteins can be

(a)
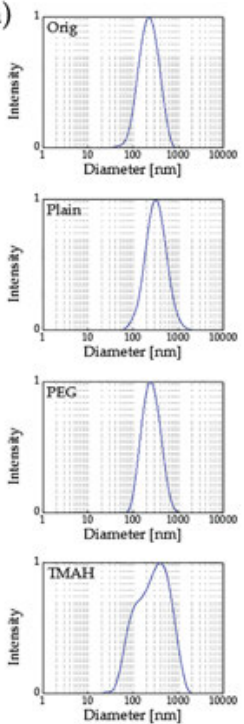

(b)

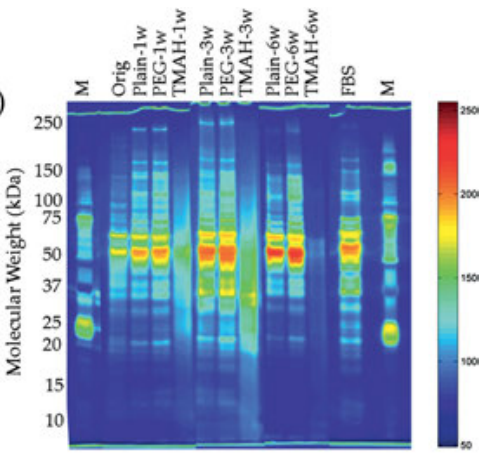

(c)

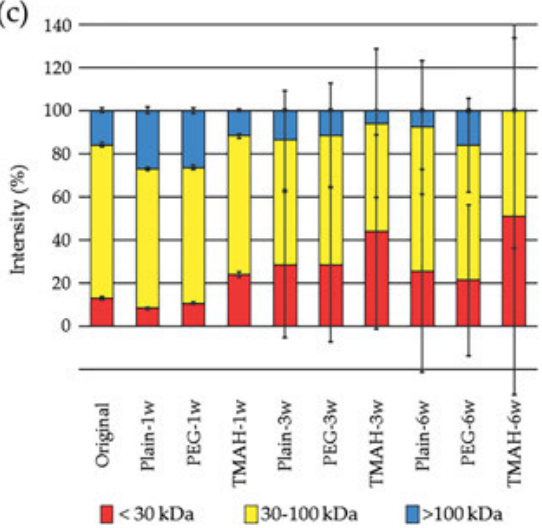

Figure 6.12: Lyophilization of biomolecule-coated nanoparticles affects the integrity of the protein corona during long-term storage. (A) After storage of the dry powders for 6 weeks the DLS measurements show that the hydrodynamic diameter of resuspended plain and polyethylene glycol (PEG) samples is very similar to that of the original sample, but for tetramethylammonium hydroxide (TMAH) an agglomeration results; (B) Proteins are degraded during lyophilization, especially in the presence of TMAH, resulting in smaller sizes after several weeks of storage demonstrated by gel electrophoresis (silver staining and false color representation); (C) Variations of the protein size ranges over the storage period (based on (B)) [57]. 
observed. The importance of this partial degradation for the storage of such particles is not clear until now.

\subsubsection{Interaction with biological systems}

\subsubsection{Cell viability studies}

To validate the biocompatibility of all used core/shell particles prior and after formation of a biomolecule corona on their surface, in vitro viability studies by using HBME cells were performed. No cytotoxic effects were confirmed for pristine MNP, PDha, and PtBAA-coated nanoparticles (polymer coatings), before and after FCS incubation, for single core as well as for multicore particles (see Figure 6.13). Similar results were found for the dextran-coated (Dex, CMD, DEAE) core/shell MNP before and after incubation in FCS.

Additionally, from the data in Figure 6.13 it can be seen that the formation of a biomolecule corona might lead to an even enhanced cell viability. Interestingly, a slight reduction in cell viability was observed for the pristine multicore MNP before FCS incubation. This effect still remains for the pristine single core MNP even after the formation of the biomolecule corona. A possible explanation for this observation might be a faster dissociation or degradation on the single core MNP with a higher surface-tovolume ratio compared to the multicore MNP.

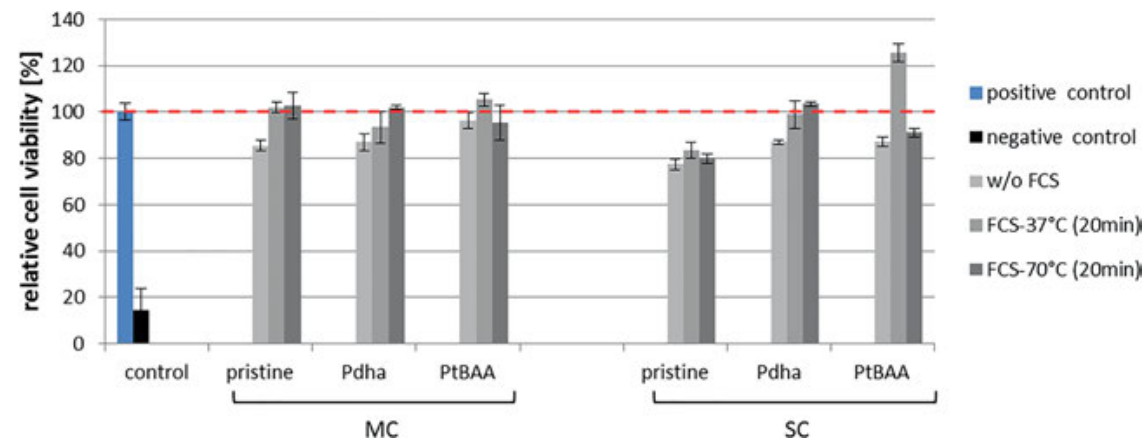

Figure 6.13: Cell viability changes in response to differently coated single core (SC) and multicore (MC) magnetic nanoparticles (MNPs) incubated at different temperatures and times. Human brain microvascular endothelial cells (HBMECs) seeded into 96-well plates in triplicate were incubated with $100 \mu \mathrm{g} / \mathrm{cm}^{2}$ (equal to $378 \mu \mathrm{g} / \mathrm{mL}$ ) of indicated particles for $24 \mathrm{~h}$. Positive controls and NCs were analogously treated with aqua bidest or cationic polyethylenimine-coated PEI-M, respectively. Detected fluorescence upon incubation with PrestoBlue reagent is normalized to positive controls [58]. 


\subsubsection{Flow cytometry}

To analyze the MNP loading to cells in dependence of a present biomolecule corona, flow cytometry was used. For this characterization method, fluorescence-labeled MNP are needed and therefore commercially available PEI-coated MNP coupled with a fluorescence dye were used as core/shell particles and incubated in FBS.

From Figure 6.14A, it becomes obvious that the intensity of MNP-associated fluorescence in and on the cells is reduced for cells incubated with MNP bearing a biomolecule corona compared to bare core/shell MNP. This can be interpreted as a reduced cellular loading for biomolecule corona-coated MNP. This means a reduced interaction of biomolecule-coated MNP with cells of about 60 and $47 \%$ compared to bare core/shell particles for incubation times of 5 and $30 \mathrm{~min}$, respectively (see Figure 6.14B). This result might be explained by an alteration of the surface potential. Due to the incubation with FCS the surface potential turns from positive to negative and the negative charge of cell membranes might hinder interactions with negatively charged nanoparticles.

\subsubsection{Confocal laser scanning microscopy}

No particle aggregation could be observed for the serum incubated PEI-coated nanoparticles.

For a more detailed investigation of this, HBMECs were incubated with MNP bearing biomolecule coronas of gradually increasing extent (by changing the FCS content of the incubation medium) and investigated by means of confocal laser scanning microscopy. Data analysis of the image stacks revealed that, the presence of a biomolecule corona reduces the particles adhesion to the cells and that the adhesion decreases with an increasing extent of the corona [50]. MATLAB analysis of the obtained stacks showed that MNP-derived signals are detectable at cells incubated with MNP of increasing biomolecule corona for 5 and $30 \mathrm{~min}$ (see Figure 6.15). It can be seen
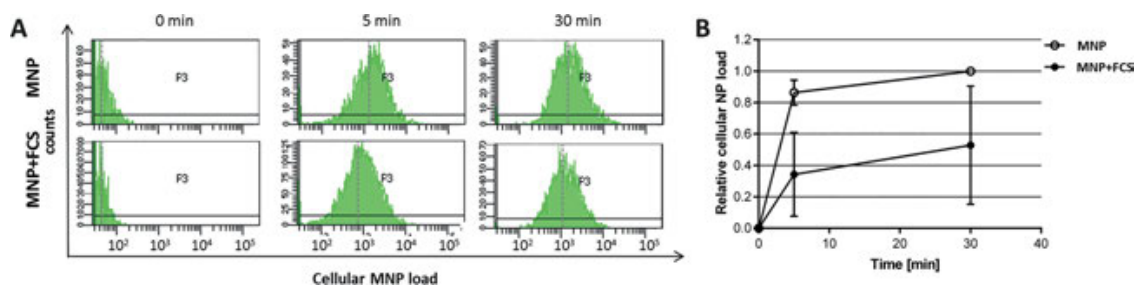

Figure 6.14: Interactions of polyethylenimine (PEI)-magnetic nanoparticles (MNPs) with human brain microvascular endothelial cells (HBMECs) as function of a present biomolecule corona. Cells were incubated for 5 or $30 \mathrm{~min}$ with particles without (MNPs) or with (MNP + FCS) a biomolecule corona. It can be seen that for both time points the presence of a biomolecule corona leads to a reduction of the MNP load to the cells [50]. 

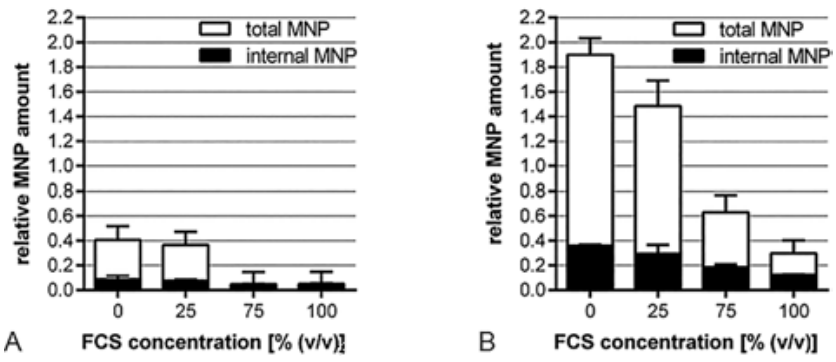

Figure 6.15: Adhesion and uptake of magnetic nanoparticles (MNPs) by human brain microvascular endothelial cells (HBMECs) depends on particles biomolecule corona. For (A) or (B) 30 min, HBMEC were incubated with $25 \mu \mathrm{g} / \mathrm{cm}^{2}$ fluorescence-labeled MNPs and imaged by means of laser scanning microscopy. Image stacks were analyzed by MATLAB where the amount of internalized MNPs was calculated from overlapping signals derived from the cytoskeletal actin and the MNP channels. Shown are means \pm standard deviation of 10-15 slices [50].

that a more pronounced biomolecule corona leads to a lower uptake of the magnetic nanohybrids into the cells for incubation times up to $30 \mathrm{~min}$. Thus, we could demonstrate that the cellular uptake and adhesion processes can be controlled by the extent of a biomolecule coating on MNP.

\subsubsection{Shell-less hen's egg model}

Furthermore, core/shell MNP and biomolecule-coated MNP were tested for their biocompatibility after systemic injection using a shell-less hen's egg model [77]. It was tested, if the prepared magnetic nanohybrids cause any adverse effects when administered to a living biological system. Another question was, if a possible irritation of the biological system can be masked with a biomolecule corona and if agglomeration of particles within the biological system can be avoided by a biomolecule coating and/or changing the particle size distribution by removal of large particles from the ensemble by means of size-dependent fractionation.

For unfractionated samples, the concentration of injected particles was adjusted to be $160 \mu \mathrm{g}_{\mathrm{MNP}} / \mathrm{mL}$. Figure 6.16 shows the probability of the occurrence of vascular lysis, hemorrhage, aggregation, and lethality for different time points (up to $24 \mathrm{~h}$ ) after injection. The validity of the assay was ensured by several controls. $0.9 \%$ sodium chloride was used as NC as recommended control by the ICCVAM [79] and sterile water as solvent control to set up the baseline for irritative effects. Sterile water showed no adverse effects.

It can be seen that the FCS-incubated CMD and PDha core/shell particles showed no aggregation and only sporadic hemorrhagic and lethal events (0-1/9 eggs) during this test comparable to the control values. The positive control, highly cationic charged PEI polymer caused a strong aggregation effect and finally a high lethality. A possible explanation for this might be the high attraction between the cationic surface of the 


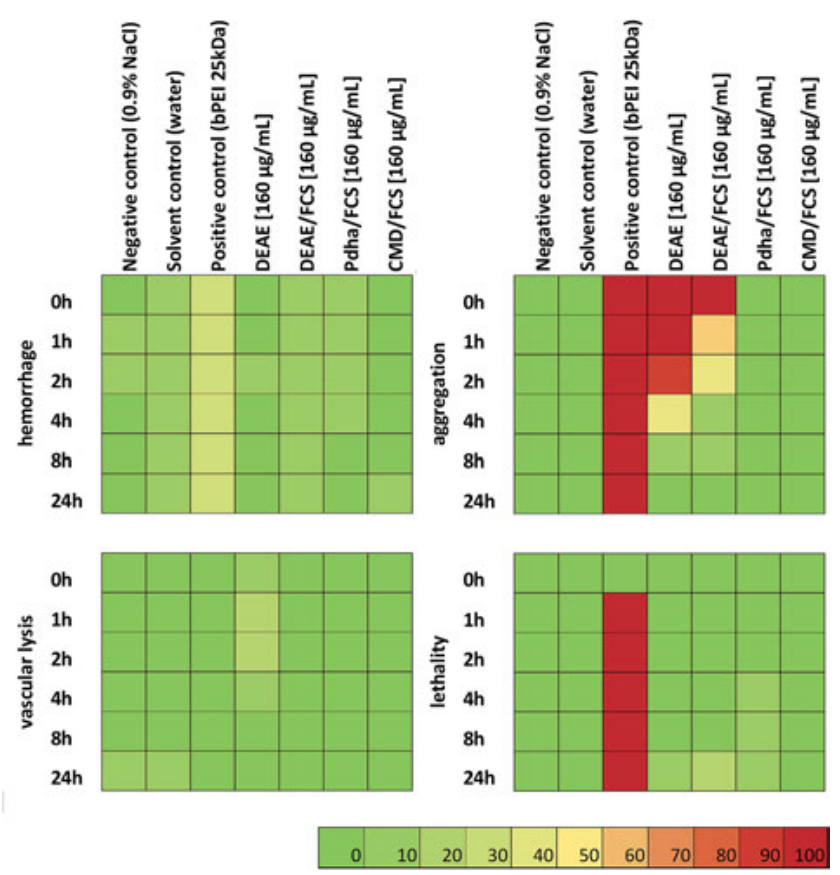

Figure 6.16: Percentual probability for the occurrence of adverse effects (aggregation, hemorrhage, vascular lysis, and lethality) for different particles and time points within the shell-less hen's egg model serving as a test to evaluate the in vivo toxicity of the magnetic nanohybrids.

polymer and anionic charged proteins present in the blood, leading to the formation of polymer/protein aggregates. For DEAE-MNP, which shows a similar surface potential like PEI, also a tendency for the occurrence of aggregation was observed. After coating these particles with biomolecules, a reduction of aggregation and a faster reversibility of the effects were obtained. Probably, the biomolecule coating leads to a less pronounced aggregate formation, because aggregates are disintegrated by shear forces of the flowing blood within the first hour after administration.

When using fractionated DEAE-MNP $\left(\mathrm{d}_{\mathrm{h}}=124 \mathrm{~nm}\right)$ instead of the previously used unfractionated ones $\left(\mathrm{d}_{\mathrm{h}}=160 \mathrm{~nm}\right)$ at a concentration of $500 \mu \mathrm{g} / \mathrm{mL}$, there is only a low tendency $(<20 \%)$ to form agglomerates for DEAE-MNP prior and after formation of the biomolecule corona. This might be explained by the removal of the larger agglomerates by centrifugation that block the blood flow in the vessels after injection (and a reduced agglomeration potential of smaller FCS-coated MNP). For this concentration, also no adverse effects were found for fractionated incubated CMD-MNP $(n=5$, data not shown). In preliminary experiments also for DEX-MNP and PEI-MNP comparable effects were observed even up to $1 \mathrm{mg} / \mathrm{mL}$ (data not shown). 
Resulting from these investigations, we were able to show that the ex ovo toxicity of our newly developed nanohybrids under the tested conditions could be highly improved by the FCS coating, as well as by the fractionation process.

\subsection{Conclusion}

The formation of a biomolecule corona is an inevitable event when nanoparticles enter biological fluids. Our investigations reveal that within seconds after bringing MNPs in contact with a protein source (FCS) a biomolecule corona is formed on the surface of the particles. This effect can be of major importance for the use of MNPs in medical applications where such particles have to be administered to the vascular system. There, the blood serves as a protein source and the biomolecule (protein) corona around the particles determines the biological fate of the particles within the body. Possibly, a precoating of the core/shell MNP with biomolecules might lead to an altered interaction of the preconditioned particles with biological systems, which can help to improve the biocompatibility of such magnetic nanohybrids.

As measured by means of MRX, DLS, and TGA, the biomolecule corona formation leads to an increase of the particle size. Due to cross-linking of the proteins on the surface of the particles, a stronger agglomeration of the biomolecule-coated MNP occurs for longer incubation times in the range from hours to days. The amount of biomolecules adsorbed to the surface was determined to be around $10 \%$ by mass of the complete magnetic nanohybrid and its surface charge is in the range from -30 to $-40 \mathrm{mV}$.

Different coatings (Dex, CMD, DEAE, PDha, and PtBAA) were used for the preparation of core/shell MNP to study the influence of surface charge on the formation of the biomolecule corona and its composition. Furthermore, the influence of incubation time and temperature on the formation was studied. Slight variations in the zeta potential of the obtained biomolecule-coated MNP are first hints for differences in amount and composition of the biomolecule corona at different incubation times and temperatures and different coating materials.

Since the formation of the biomolecule corona is a very complex process, which is influenced by a lot of different factors, it is not possible to derive a general model for the corona formation for all used coatings. For the most of the here tested core/shell MNP, our TGA results show that at lower temperatures $\left(25\right.$ and $\left.37^{\circ} \mathrm{C}\right)$ the amount of biomolecules does not increase with increasing incubation time whereas this is the case at elevated temperatures $\left(50\right.$ and $70^{\circ} \mathrm{C}$ ). In that regard, polyzwitterionic PDha is a highly interesting material for coating of MNP since a distinctly lower amount of biomolecules was adsorbed during the FCS incubation compared to all other coating materials. The amount of biomolecules adsorbed to the surface of the core/shell MNPs can be tuned by the composition of the incubation medium. A higher amount of biomolecules in the 
incubation medium leads to an increasing volume of the biomolecule corona and thus a larger thickness of the biomolecule coating.

A semi-quantitative analysis of the gels obtained from SDS-PAGE confirmed the results from TGA and provided additional information about the composition of the proteins contributing to the formed biomolecule corona. Although for all coating materials and temperatures an influence on the ratios between low-molecular-weight proteins $(<25 \mathrm{kDa})$, medium-molecular-weight proteins $(25-100 \mathrm{kDa})$, and highmolecular-weight $(>100 \mathrm{kDa})$ proteins was found, no general trends can be derived from the obtained data so far.

Before application of such particles to biological systems it has to be assured that the particle preparations are free from pathogens and also procedures have to be established, which enable the long-term storage of the particles.

For the sterilization by means of UV radiation, we found no damaging effects for biomolecule-coated MNP. Contrary, the treatment of biomolecule-coated particles by means of autoclaving leads to significant changes in the integrity of the proteins and thus this method is not suitable for sterilization. Freezing at -15 and $-80{ }^{\circ} \mathrm{C}$ allows storage for up to 1 week. After that, a remarkable degradation of the larger proteins in combination with an agglomeration of the particles takes place. During lyophilization, the addition of PEG to the samples revealed well dispersed fluids after resuspension of the powders. For storage times longer than one week, a slight degradation of the proteins was observed. This storage period might be extended by using optimized storing conditions (e.g., lower temperature, oxygen exclusion, reduced humidity). Furthermore, it has to be investigated, which consequences this slight degradation for the application of such particles to biological systems means. Therefore, future studies are needed to elucidate the treatment-dependent changes on the biomolecule corona, as well as on the single protein level with regard to cytotoxicity and immunogenicity. In conclusion, we state that the UV sterilization followed by lyophilization under addition of PEG is the most promising route for the preparation of sterilized long-term durable biomolecule-coated MNP.

In cell viability tests, no cytotoxicity was found for all tested biomolecule-coated MNP. A higher amount of biomolecules adsorbed to the surface lead to an increased cell viability. By flow cytometry, it was shown that the presence of a biomolecule corona reduces the interaction of nanoparticles with cells during short-term incubation in the range from 5 to $30 \mathrm{~min}$. Laser scanning microscopy revealed that a more pronounced biomolecule corona leads to a retarded uptake of the magnetic nanohybrids into the cells. Thus, we could demonstrate that for short-term incubation the cellular uptake and adhesion processes can be controlled by the extent of a biomolecule coating on MNP. Nonetheless, possible effects of long-term incubation have to be investigated. By using a shell-less hen's egg model, no adverse effects of all biomolecule-coated MNP for this in vivo/ex ovo test were found. Resulting from these investigations, we were able to show that the in vivo biocompatibility of our newly developed nanohybrids under the tested conditions could be highly improved by the biomolecule coating. 
In summary, we developed and described a novel type of core/shell nanoparticles with improved biocompatibility for medical application. These magnetic nanohybrids bear a second coating of biomolecules, which enables the control of the interaction of these particles with biological systems. The biomolecule corona may mask adverse effects on biological systems of cytotoxic core/shell MNP. In ongoing studies, the biocompatibility has to be tested for mammals and the consequences of variations in the composition of the biomolecule corona for the application have to be investigated in more detail.

Acknowledgments: This work was supported by the Deutsche Forschungsgemeinschaft (DFG) in the framework of SPP 1681 (FKZ: CL202/3-1, CL202/3-2, CL202/3-3, DU1293/4-1, DU1293/4-2, DU1293/7-3, and SCHA1640/7-1). The authors thank all partners within the SPP 1681 for the fruitful collaboration and especially Prof. Stefan Odenbach (TU Dresden) for the excellent coordination of the DFG Priority Programme.

Figures 6.7, 6.8, 6.14, and 6.15 are reprinted from "Gräfe, C.; Weidner, A.; von der Lühe, M.; Bergemann, C.; Schacher, F.H.; Clement, J.H.; Dutz, S. Intentional formation of a protein corona on nanoparticles: Serum concentration affects protein corona mass, surface charge, and nanoparticle-cell interaction. Int. J. Biochem. Cell Biol. 75: 196-202 (2016)", with permission from Elsevier.

Figures 6.9, 6.10, and 6.13 are reprinted from "Gräfe, C.; von der Lühe, M.; Weidner, A.; Globig, P.; Clement, J.H.; Dutz, S.; Schacher, F.H. Protein corona formation and its constitutional changes on magnetic nanoparticles in serum featuring a polydehydroalanine coating: effects of charge and incubation conditions. Nanotechnology 30: 265707 (2019)”, (C) IOP Publishing. Reproduced with permission. All rights reserved.

Author contribution: All the authors have accepted responsibility for the entire content of this submitted manuscript and approved submission.

Research funding: Deutsche Forschungsgemeinschaft (DFG) in the framework of SPP 1681 (FKZ: CL202/3-1, CL202/3-2, CL202/3-3, DU1293/4-1, DU1293/4-2, DU1293/7-3, and SCHA1640/7-1).

Conflict of interest statement: The authors declare no conflicts of interest regarding this article.

\section{References}

1. Cardoso VF, Francesko A, Ribeiro C, Banobre-Lopez M, Martins P, Lanceros-Mendez S. Advances in magnetic nanoparticles for biomedical applications. Adv Healthc Mater 2018;7. https://doi.org/10. 1002/adhm.201700845.

2. Krishnan KM. Biomedical nanomagnetics: a spin through possibilities in imaging, diagnostics, and therapy. IEEE Trans Magn 2010;46:2523-58. 
3. Pankhurst QA, Connolly J, Jones SK, Dobson J. Applications of magnetic nanoparticles in biomedicine. J Phys D Appl Phys 2003;36:R167-81.

4. Wu K, Su DQ, Liu JM, Saha R, Wang JP. Magnetic nanoparticles in nanomedicine: a review of recent advances. Nanotechnology 2019;30. https://doi.org/10.1088/1361-6528/ab4241.

5. Dutz S, Hergt R. Magnetic particle hyperthermia-a promising tumour therapy?. Nanotechnology 2014;25. https://doi.org/10.1088/0957-4484/25/45/452001.

6. Gilchrist RK, Medal R, Shorey WD, Hanselman RC, Parrott JC, Taylor CB. Selective inductive heating of lymph nodes. Ann Surg 1957;146:596-606.

7. Perigo EA, Hemery G, Sandre O, Ortega D, Garaio E, Plazaola F, et al. Fundamentals and advances in magnetic hyperthermia. Appl Phys Rev 2015;2. https://doi.org/10.1063/1.4935688.

8. Estelrich J, Escribano E, Queralt J, Busquets MA. Iron oxide nanoparticles for magnetically-guided and magnetically-responsive drug delivery. Int J Mol Sci 2015;16:8070-101.

9. Lubbe AS, Bergemann C, Riess H, Schriever F, Reichardt P, Possinger K, et al. Clinical experiences with magnetic drag targeting: a phase I study with 4'-epidoxorubicin in 14 patients with advanced solid tumors. Cancer Res 1996;56:4686-93.

10. Gleich B, Weizenecker R. Tomographic imaging using the nonlinear response of magnetic particles. Nature 2005;435:1214-7.

11. Dadfar SM, Roemhild K, Drude NI, von Stillfried S, Knuchel R, Kiessling F, et al. Iron oxide nanoparticles: diagnostic, therapeutic and theranostic applications. Adv Drug Deliv Rev 2019;138: 302-25.

12. Wu W, Jiang CZ, Roy VAL. Designed synthesis and surface engineering strategies of magnetic iron oxide nanoparticles for biomedical applications. Nanoscale 2016;8:19421-74.

13. Wu W, Wu ZH, Yu T, Jiang CZ, Kim WS. Recent progress on magnetic iron oxide nanoparticles: synthesis, surface functional strategies and biomedical applications. Sci Technol Adv Mater 2015; 16. https://doi.org/10.1088/1468-6996/16/2/023501.

14. Dutz S. Are magnetic multicore nanoparticles promising candidates for biomedical applications?. IEEE Trans Magn 2016;52. https://doi.org/10.1109/tmag.2016.2570745.

15. Dutz S, Kettering M, Hilger I, Muller R, Zeisberger M. Magnetic multicore nanoparticles for hyperthermia-influence of particle immobilization in tumour tissue on magnetic properties. Nanotechnology 2011;22:265102.

16. Kostopoulou A, Brintakis K, Fragogeorgi E, Anthousi A, Manna L, Begin-Colin S, et al. Iron oxide colloidal nanoclusters as theranostic vehicles and their interactions at the cellular level. Nanomaterials 2018;8. https://doi.org/10.3390/nano8050315.

17. Lartigue L, Hugounenq P, Alloyeau D, Clarke SP, Levy M, Bacri J-C, et al. Cooperative organization in iron oxide multi-core nanoparticles potentiates their efficiency as heating mediators and MRI contrast agents. ACS Nano 2012;6:10935-49.

18. Kratz H, Taupitz M, de Schellenberger AA, Kosch O, Eberbeck D, Wagner S, et al. Novel magnetic multicore nanoparticles designed for MPI and other biomedical applications: from synthesis to first in vivo studies. PloS One 2018;13. https://doi.org/10.1371/journal.pone.0190214.

19. Del Pino P, Pelaz B, Zhang Q, Maffre P, Nienhaus GU, Parak WJ. Protein corona formation around nanoparticles - from the past to the future. Mater Horiz 2014;1:301-13.

20. Tenzer S, Docter D, Rosfa S, Wlodarski A, Kuharev J, Rekik A, et al. Nanoparticle size is a critical physicochemical determinant of the human blood plasma corona: a comprehensive quantitative proteomic analysis. ACS Nano 2011;5:7155-67.

21. Vroman L. Effect of adsorbed proteins on the wettability of hydrophilic and hydrophobic solids. Nature 1962;196:476-7.

22. Nel AE, Maedler L, Velegol D, Xia T, Hoek EMV, Somasundaran P, et al. Understanding biophysicochemical interactions at the nano-bio interface. Nat Mater 2009;8:543-57. 
23. Dai Q, Bertleff-Zieschang N, Braunger JA, Bjornmalm M, Cortez-Jugo C, Caruso F. Particle targeting in complex biological media. Adv Healthc Mater 2018;7. https://doi.org/10.1002/adhm. 201700575.

24. Eberbeck D, Kettering M, Bergemann C, Zirpel P, Hilger I, Trahms L. Quantification of the aggregation of magnetic nanoparticles with different polymeric coatings in cell culture medium. J Phys D Appl Phys 2010;43. https://doi.org/10.1088/0022-3727/43/40/405002.

25. Illes E, Szekeres M, Toth IY, Farkas K, Foldesi I, Szabo A, et al. PEGylation of superparamagnetic iron oxide nanoparticles with self-organizing polyacrylate-PEG brushes for contrast enhancement in MRI diagnosis. Nanomaterials 2018;8. https://doi.org/10.3390/nano8100776.

26. Jain P, Pawar RS, Pandey RS, Madan J, Pawar S, Lakshmi PK, et al. In-vitro in-vivo correlation (IVIVC) in nanomedicine: is protein corona the missing link?. Biotechnol Adv 2017;35: 889-904.

27. Mahmoudi M, Lynch I, Ejtehadi MR, Monopoli MP, Bombelli FB, LaurentS. et al. Proteinnanoparticle interactions: opportunities and challenges. Chem Rev 2011;111:5610-37.

28. Boraschi D, Italiani P, Palomba R, Decuzzi P, Duschl A, Fadeel B, et al. Nanoparticles and innate immunity: new perspectives on host defence. Semin Immunol 2017;34:33-51.

29. Lynch I, Dawson KA. Protein-nanoparticle interactions. Nano Today 2008;3:40-7.

30. Pearson RM, Juettner VV, Hong S. Biomolecular corona on nanoparticles: a survey of recent literature and its implications in targeted drug delivery. Front Chem 2014;2:108-108.

31. Winzen S, Schoettler S, Baier G, Rosenauer C, Mailaender V, Landfester K, et al. Complementary analysis of the hard and soft protein corona: sample preparation critically effects corona composition. Nanoscale 2015;7:2992-3001.

32. Cedervall T, Lynch I, Lindman S, Berggard T, Thulin E, Nilsson H, et al. Understanding the nanoparticle-protein corona using methods to quantify exchange rates and affinities of proteins for nanoparticles. Proc Natl Acad Sci U S A 2007;104:2050-5.

33. Pederzoli F, Tosi G, Vandelli MA, Belletti D, Forni F, Ruozi BProtein corona and nanoparticles: how can we investigate on? Wiley Interdiscip Rev Nanomed Nanobiotechnol 2017;9. https://doi.org/ 10.1002/wnan.1467.

34. Weber C, Morsbach S, Landfester K. Possibilities and limitations of different separation techniques for the analysis of the protein corona. Angew Chem Int Ed 2019;58:12787-94.

35. Brancolini G, Bellucci L, Maschio MC, Di Felice R, Corni S. The interaction of peptides and proteins with nanostructures surfaces: a challenge for nanoscience. Curr Opin Colloid Interface Sci 2019; 41:86-94.

36. Langevin D, Lozano O, Salvati A, Kestens V, Monopoli M, Raspaud E, et al. Inter-laboratory comparison of nanoparticle size measurements using dynamic light scattering and differential centrifugal sedimentation. Nanoimpact 2018;10:97-107.

37. Nel AE, Madler L, Velegol D, Xia T, Hoek EMV, Somasundaran P, et al. Understanding biophysicochemical interactions at the nano-bio interface. Nat Mater 2009;8:543-57.

38. Roecker C, Poetzl M, Zhang F, Parak WJ, Nienhaus GU. A quantitative fluorescence study of protein monolayer formation on colloidal nanoparticles. Nat Nanotechnol 2009;4:577-80.

39. Bohorquez AC, Unni M, Belsare S, Chiu-Lam A, Rice L, Pampo C, et al. Stability and mobility of magnetic nanoparticles in biological environments determined from dynamic magnetic susceptibility measurements. Bioconjug. Chem. 2018;29:2793-805.

40. Eberbeck D, Wiekhorst F, Steinhoff U, Trahms L. Quantification of biomolecule agglutination by magnetorelaxometry. Appl Phys Lett 2009;95. https://doi.org/10.1063/1.3267054.

41. Soukup D, Moise S, Cespedes E, Dobson J, Telling ND. In situ measurement of magnetization relaxation of internalized nanoparticles in live cells. ACS Nano 2015;9:231-40.

42. Bekdemir A, Liao SY, Stellacci F. On the effect of ligand shell heterogeneity on nanoparticle/ protein binding thermodynamics. Colloids Surf B Biointerfaces 2019;174:367-73. 
43. Casals E, Pfaller T, Duschl A, Oostingh GJ, Puntes V. Time evolution of the nanoparticle protein corona. ACS Nano 2010;4:3623-32.

44. Huhn D, Kantner K, Geidel C, Brandholt S, De Cock I, Soenen SJH, et al. Polymer-coated nanoparticles interacting with proteins and cells: focusing on the sign of the net charge. ACS Nano 2013;7:3253-63.

45. Kumar S, Yadav I, Aswal VK, Kohlbrecher J. Structure and interaction of nanoparticle-protein complexes. Langmuir 2018;34:5679-95.

46. Lundqvist M, Stigler J, Elia G, Lynch I, Cedervall T, Dawson KA. Nanoparticle size and surface properties determine the protein corona with possible implications for biological impacts. Proc Natl Acad Sci U S A 2008;105:14265-70.

47. Mahmoudi M, Abdelmonem AM, Behzadi S, Clement JH, Dutz S, Ejtehadi MR, et al. Temperature: the "ignored" factor at the NanoBio interface. ACS Nano 2013;7:6555-62.

48. Partikel K, Korte R, Mulac D, Humpf HU, Langer K. Serum type and concentration both affect the protein-corona composition of PLGA nanoparticles. Beilstein J Nanotechnol 2019;10: 1002-15.

49. Bolanos K, Kogan MJ, Araya E. Capping gold nanoparticles with albumin to improve their biomedical properties. Int J Nanomed 2019;14:6387-406.

50. Grafe C, Weidner A, von der Luhe M, Bergemann C, Schacher FH, Clement JH, et al. Intentional formation of a protein corona on nanoparticles: serum concentration affects protein corona mass, surface charge, and nanoparticle-cell interaction. Int J Biochem Cell Biol 2016;75:196-202.

51. Gulati NM, Stewart PL, Steinmetz NF. Bioinspired shielding strategies for nanoparticle drug delivery applications. Mol Pharm 2018;15:2900-9.

52. Mariam J, Sivakami S, Dongre PM. Albumin corona on nanoparticles - a strategic approach in drug delivery. Drug Deliv 2016;23:2668-76.

53. Monteiro-Riviere NA, Samberg ME, Oldenburg SJ, Riviere JE. Protein binding modulates the cellular uptake of silver nanoparticles into human cells: implications for in vitro to in vivo extrapolations?. Toxicol Lett 2013;220:286-93.

54. Schottler S, Becker G, Winzen S, Steinbach T, Mohr K, Landfester K, et al. Protein adsorption is required for stealth effect of poly(ethylene glycol)-and poly(phosphoester)-coated nanocarriers. Nat Nanotechnol 2016;11:372-7.

55. Biehl P, von der Luhe M, Dutz S, Schacher FH. Synthesis, characterization, and applications of magnetic nanoparticles featuring polyzwitterionic coatings. Polymers 2018;10. https://doi.org/ 10.3390/polym10010091.

56. Billing M, Grafe C, Saal A, Biehl P, Clement JH, Dutz S, et al. Zwitterionic iron oxide (-Fe2O3) nanoparticles based on P(2VP-grad-AA) copolymers. Macromol Rapid Commun 2017;38. https:// doi.org/10.1002/marc.201600637.

57. Dutz S, Wojahn S, Grafe C, Weidner A, Clement JH. Influence of sterilization and preservation procedures on the integrity of serum protein-coated magnetic nanoparticles. Nanomaterials 2017; 7. https://doi.org/10.3390/nano7120453.

58. Grafe C, von der Luhe M, Weidner A, Globig P, Clement JH, Dutz S, et al. Protein corona formation and its constitutional changes on magnetic nanoparticles in serum featuring a polydehydroalanine coating: effects of charge and incubation conditions. Nanotechnology 2019; 30. $h$ ttps://doi.org/10.1088/1361-6528/abOedo.

59. Muller EK, Grafe C, Wiekhorst F, Bergemann C, Weidner A, Dutz S, et al. Magnetic nanoparticles interact and pass an in vitro co-culture blood-placenta barrier model. Nanomaterials 2018;8. https://doi.org/10.3390/nano8020108.

60. von der Luhe M, Gunther U, Weidner A, Grafe C, Clement JH, Dutz S, et al. SPION@polydehydroalanine hybrid particles. Rsc Adv 2015;5:31920-9. 
61. von der Luhe M, Weidner A, Dutz S, Schacher FH. Reversible electrostatic adsorption of polyelectrolytes and bovine serum albumin onto polyzwitterion-coated magnetic multicore nanoparticles: implications for sensing and drug delivery. ACS Appl Nano Mater 2018;1:232-44.

62. Weidner A, Gräfe C, Von der Luhe M, Remmer H, Clement JH, Eberbeck D, et al. Preparation of coreshell hybrid materials by producing a protein corona around magnetic nanoparticles. Nanoscale Res Lett 2015;10:992.

63. Billing M, Festag G, Bellstedt P, SchacherAmphiphilic FH. And double hydrophilic block copolymers containing a polydehydroalanine block. Polym Chem 2017;8:936-45.

64. Guenther U, Sigolaeva LV, Pergushov DV, Schacher FH. Polyelectrolytes with tunable charge based on polydehydroalanine: synthesis and solution properties. Macromol Chem Phys 2013;214: 2202-12.

65. Khalafalla SE, Reimers GW. Preparation of dilution-stable aqueous magnetic fluids. IEEE Trans Magn 1980;16:178-83.

66. Dutz S, Andrae W, Hergt R, Mueller R, Oestreich C, Schmidt C, et al. Influence of dextran coating on the magnetic behaviour of iron oxide nanoparticles. J Magn Magn Mater 2007;311:51-4.

67. Dutz S, Clement JH, Eberbeck D, Gelbrich T, Hergt R, Mueller R, et al. Ferrofluids of magnetic multicore nanoparticles for biomedical applications. J Magn Magn Mater 2009;321:1501-4.

68. Hergt R, Dutz S, Mueller R, Zeisberger M. Magnetic particle hyperthermia: nanoparticle magnetism and materials development for cancer therapy. J Phys Condens Matter 2006;18:S2919-34.

69. Suzdalev IP, Maksimov YV, Buravtsev VN, Imshennik VK, Kazakevich AG, Novichikhin SV. The formation and properties of a system of iron oxide nanoclusters. Colloid J 2000;62:224-33.

70. Bodenschatz W. Handbuch für den Desinfektor in Ausbildung und Praxis. München/Germany: Elsevier; 1993.

71. Kurzhals H. Kühlen und Gefrieren von Lebensmitteln. Hamburg/Germany: BEHRS Verlag; 2007.

72. Wang W. Lyophilization and development of solid protein pharmaceuticals. Int J Pharm 2000;203: $1-60$.

73. Dutz S, Hergt R. The role of interactions in systems of single domain ferrimagnetic iron oxide nanoparticles. J. Nano- Electron. Phys. 2012;4:20101-7.

74. Dutz S, Hergt R, Murbe J, Topfer J, Muller R, Zeisberger M, et al. Magnetic nanoparticles for biomedical heating applications. Z Phys Chem 2006;220:145-51.

75. Schneider CA, Rasband WS, Eliceiri KW. NIH Image to Image): 25 years of image analysis. Nat Methods 2012;9:671-5.

76. Ludwig F, Mauselein S, Heim E, Schilling M. Magnetorelaxometry of magnetic nanoparticles in magnetically unshielded environment utilizing a differential fluxgate arrangement. Rev Sci Instrum 2005;76. https://doi.org/10.1063/1.2069776.

77. Schlenk F, Werner S, Rabel M, Jacobs F, Bergemann C, Clement JH, et al. Comprehensive analysis of the in vitro and ex ovo hemocompatibility of surface engineered iron oxide nanoparticles for biomedical applications. Arch Toxicol 2017;91:3271-86.

78. Hamburger V, Hamilton HL. A series of normal stages in the development of the chick embryo. J Morphol 1951;88:49\&\#x2013.

79. NIH. In vitro cytotoxicity test methods for estimating starting doses for acute oral systemic toxicity testing ICCVAM test method evaluation report 2006. NIH Publication No. 07-4519.

80. Chantrell RW, Popplewell J, Charles SW. Measurements of particle-size distribution parameters in ferrofluids. IEEE Trans Magn 1978;14:975-7.

81. BiehI P, von der Luhe M, Schacher FH. Reversible adsorption of methylene blue as cationic model cargo onto polyzwitterionic magnetic nanoparticles. Macromol Rapid Commun 2018;39. https:// doi.org/10.1002/marc.201800017.

82. Fasold $\mathrm{H}$, Klappenberger J, Meyer $\mathrm{C}$, Remold $\mathrm{H}$. Bifunctional reagents for the crosslinking of proteins. Angew Chem Int Ed 1971;10:795. 